JOURNAL OF THE

AMERICAN MATHEMATICAL SOCIETY

Volume 26, Number 3, July 2013, Pages 853-877

S 0894-0347(2013)00762-6

Article electronically published on March 18, 2013

\title{
LOCAL INDECOMPOSABILITY OF TATE MODULES \\ OF NON-CM ABELIAN VARIETIES WITH REAL MULTIPLICATION
}

\author{
HARUZO HIDA
}

\section{CONTENTS}

1. Deformation space of an AVRM

2. Hilbert modular Shimura variety

3. Eigendifferentials

4. Eigencoordinates

5. Local indecomposability of an AVRM

6. CM periods and proportionality constants

7. Density of partially ordinary primes

Acknowledgement

References

As quoted by Ghate-Vatsal in GV], Question 1 (and answered there affirmatively to a good extent), it is a fundamental problem posed by R. Greenberg to decide indecomposability of an elliptic modular $p$-adic Galois representation restricted to the decomposition group at $p$ in the ordinary case (as long as the representation is not of CM type). Jointly with B. Balasubramanyam, they have generalized the result to the cases of Hilbert modular forms for primes $p$ splitting completely in the totally real base field (see GV1]). There are some good applications of their results (see for example $[\mathrm{E}]$ and $[\mathrm{G}]$ ). We can ask the same question for $p$-adic Galois representations arising from the Tate module of an abelian variety $A$ with real multiplication defined over a number field $k$; so, $\operatorname{End}\left(A_{/ k}\right)$ contains the integer ring $O$ of a totally real field of $\operatorname{degree} \operatorname{dim} A$. This concerns the $\mathfrak{p}$-adic Tate module $T_{\mathfrak{p}} A$ for a prime $\mathfrak{p} \mid(p)$ of $O$ as a module over the decomposition group $D_{p}$ at a place $\mathfrak{P}$ over $p$ of $k$. Suppose that $A$ has good reduction modulo $\mathfrak{P}$. If the finite flat group scheme $A[\mathfrak{p}]$ of $\mathfrak{p}$-torsion points is local-local at $\mathfrak{P}$, as is well known, the $D_{p}$-module $A[\mathfrak{p}]\left(\overline{\mathbb{Q}}_{p}\right)$ or $T_{\mathfrak{p}} A \otimes_{O_{\mathfrak{p}}} F_{\mathfrak{p}}$ is often irreducible (as proven in [ALR], IV-38, $\S$ A.2.2 for elliptic curves; see also the generalization of Serre's modulo $p$ modularity conjecture for totally real $k$; in particular, $\S 3.1$ of [BDJ]). In this paper, we limit

Received by the editors April 1, 2012 and, in revised form, December 27, 2012.

2010 Mathematics Subject Classification. Primary 14G35, 11G15, 11G18, 11F80; Secondary $11 \mathrm{G} 10,14 \mathrm{~L} 05$

Key words and phrases. Abelian variety, Tate module, real/complex multiplication, Galois representation, deformation.

The author is partially supported by NSF grants DMS 0753991 and DMS 0854949. 
ourselves to the case where $A[\mathfrak{p}]\left(\overline{\mathbb{F}}_{p}\right) \neq\{0\}$. This assumption does not necessarily mean that $A$ has ordinary good reduction at $p$.

We answer this question affirmatively in Theorem 5.1 for an abelian variety with sufficiently many real multiplications but without complex multiplication (over $\overline{\mathbb{Q}}$ ) if the endomorphism totally real field $F$ and the field $k$ of definition are unramified at $p$ over $\mathbb{Q}$. Since simple factors of the jacobian of a Shimura curve are often of this type, we should be able to answer the original question by Greenberg. Indeed, Bin Zhao [Z has just removed our assumption of the unramifiedness of $p$ in the base field $k$ and in the multiplication field $F$ and has proved unconditionally the indecomposability for the nearly ordinary Galois representation of each weight 2 Hilbert cusp form at least over an odd degree totally real base field. If the base field has even degree, he had to assume square-integrability at a finite place or the existence of an abelian variety associated to the cusp form.

Since a CM abelian variety with ordinary good reduction at $p$ has semi-simple Tate module over $D_{p}$, we may regard the $p$-local indecomposability of the $\mathfrak{p}$-adic Tate module as a characterization of non-CM/CM abelian varieties via local $p$ inertia action. The importance of such characterization is emphasized in [H11a], and other examples of characterization are listed there.

Throughout this paper, we fix an algebraic closure $\overline{\mathbb{Q}}$ in $\mathbb{C}$ and an algebraic closure $\overline{\mathbb{Q}}_{v}$ of $\mathbb{Q}_{v}$ (the $v$-adic field or $\mathbb{R}$ for $v=\infty$ ) for each rational place $v$. We write $\mathbb{C}_{v}$ for the completion of $\overline{\mathbb{Q}}_{v}$ under $v$. Therefore, for each $v$, we fix a field embedding $i_{v}: \overline{\mathbb{Q}} \hookrightarrow \mathbb{C}_{v}$ (here $i_{\infty}$ coincides with the original inclusion $\overline{\mathbb{Q}} \subset \mathbb{C}$ ) and identify all $\mathbb{C}_{v}$ with $\mathbb{C}$ so that we have the following commutative diagram for each $v$ :

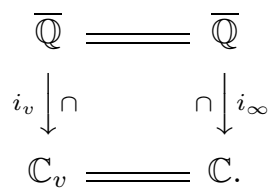

Here is a sketch of our proof of indecomposability (and an outline of the paper), assuming for simplicity that the abelian variety $A$ over a number field $k \subset \overline{\mathbb{Q}}$ has multiplication by the integer ring $O$ of a real quadratic field $F$; so, $2=[F: \mathbb{Q}]=$ $\operatorname{dim} A$ and $\operatorname{End}_{F}^{0}\left(A_{/ \overline{\mathbb{Q}}}\right)=\operatorname{End}_{F}^{0}\left(A_{/ k} \times_{k} \overline{\mathbb{Q}}_{/ \overline{\mathbb{Q}}}\right)=F$ (this means that $A$ does not have complex multiplication), where " $\operatorname{End}_{F}^{0}(?)$ " denotes the total quotient ring of the $O$-linear endomorphism algebra $\operatorname{End}_{O}(?)$ of the object "?" inside. Such abelian varieties are parameterized by Hilbert modular varieties whose theory we recall in Section 2 to the extent we need (explicitly or implicitly).

Let $\mathfrak{O}$ denote the integer ring of $k$. We denote primes of $k$ by uppercase Gothic letters and those of $F$ by lowercase Gothic letters, and the corresponding Roman (lowercase) character is the residual characteristic of a prime denoted by a Gothic character. Suppose further for simplicity that $A$ has ordinary good reduction at primes $\mathfrak{P}$ and $\mathfrak{L}$ with $p \neq l$. Contrary to what we want to prove, suppose that $A\left[\mathfrak{p}^{\infty}\right]$ for a prime $\mathfrak{p}$ above $p$ has a semi-simple Tate module over $D_{p}=\operatorname{Gal}\left(\overline{\mathbb{Q}}_{p} / k_{\mathfrak{P}}\right)$. Then the $\mathfrak{p}$-adic Serre-Tate coordinate $t_{\mathfrak{p}}(A)$ of $A$ is equal to 1 . If $\mathfrak{p}$ is a unique prime over $(p)$ in $O$, this identity $t_{\mathfrak{p}}(A)=1$ forces $A$ to be a canonical lift having complex multiplication. Thus we assume $(p)=\mathfrak{p p}^{\prime}$ in $F$ with $\mathfrak{p} \neq \mathfrak{p}^{\prime}$. By the Serre-Tate theory, for the Witt vector ring $W_{p}$ with coefficients in an algebraic closure $\overline{\mathbb{F}}_{p}$ of $\mathbb{F}_{p}$, the deformation space of the reduction $A_{\mathfrak{P}}=A \otimes_{\mathfrak{O}_{\mathfrak{P}}} \overline{\mathbb{F}}_{p}$ (for $\mathbb{F}_{\mathfrak{P}}:=\mathfrak{O} / \mathfrak{P}$ 
regarded as sitting in $\overline{\mathbb{F}}_{p}$ ) is isomorphic to $\widehat{S}_{/ W_{p}}=\widehat{S}_{p / W_{p}}:=\widehat{\mathbb{G}}_{m} \otimes_{\mathbb{Z}_{p}} O_{p}=\widehat{S}_{\mathfrak{p}} \times \widehat{S}_{\mathfrak{p}^{\prime}}$ with $\widehat{S}_{\mathfrak{p}}:=\widehat{\mathbb{G}}_{m} \otimes_{\mathbb{Z}_{p}} O_{\mathfrak{p}}$, and $t_{\mathfrak{p}}$ is the coordinate of the multiplicative formal group $\widehat{\mathbb{G}}_{m} \otimes_{\mathbb{Z}_{p}} O_{\mathfrak{p}}=\widehat{\mathbb{G}}_{m}\left(\right.$ as $\left.O_{\mathfrak{p}}=\mathbb{Z}_{p}\right)$. In other words, $\widehat{\mathbb{G}}_{m / W_{p}}$ is the formal completion of $\mathbb{G}_{m}=\operatorname{Spec}\left(W_{p}\left[t_{\mathfrak{p}}, t_{\mathfrak{p}}^{-1}\right]\right)$ along the origin (i.e., the identity) of its reduction modulo $p$. We recall in Section 1 some details of the construction of Serre-Tate coordinates.

The field $M=\operatorname{End}_{F}^{0}\left(A_{\mathfrak{P} / \overline{\mathbb{F}}_{p}}\right)$ is a CM quadratic extension of $F$. We have two Serre-Tate coordinates, $t_{\mathfrak{p}}$ and $t_{\mathfrak{p}^{\prime}}$. Since $A$ does not have CM, we must have $t_{\mathfrak{p}^{\prime}}(A) \neq 1$ (as the origin of $\widehat{S}$ corresponds to the canonical lift of $A_{\mathfrak{P}}$ which has complex multiplication by the CM field $M)$. By the universality of $\widehat{S}, M^{\times}$acts on $\widehat{S}$ naturally by an isogeny action. Then by the reciprocity law of $\widehat{S}$ in [H10], $\S 3.3$, we have $t_{\mathfrak{p}} \circ \alpha=t_{\mathfrak{p}}^{\alpha^{\sigma(1-c)}}$ for an embedding $\sigma: M \hookrightarrow \overline{\mathbb{Q}}$ inducing the place $\mathfrak{p}$ of $F$ after composing with $i_{p}$. Here $c$ denotes the generator of $\operatorname{Gal}(M / F)$. For the $l$-adic place $\mathfrak{l}$ induced by $i_{l} \circ \sigma$, we have the Serre-Tate coordinate $t_{\mathfrak{l}}$ of the deformation space $\widehat{S}_{\mathfrak{l}}=\widehat{\mathbb{G}}_{m} \otimes_{\mathbb{Z}_{p}} O_{\mathfrak{l}}$ of $A_{\mathfrak{L}}$. Now the abelian variety $A$ gives rise to a $k$-point $x$ of the Hilbert modular Shimura variety $S h_{/ \mathbb{Q}}$ for $G L(2)_{/ F}$. Looking at the $k$-tangent space $\operatorname{Tan}_{x}$ at $x \in S h_{K}(k)$ (for a neat open compact subgroup $K \subset G L_{2}\left(F_{\mathbb{A}}^{(\infty)}\right)$ maximal at $p$ and $\left.l\right)$, the Lie algebras $\operatorname{Lie}\left(\widehat{S}_{\mathfrak{p}}\right)$ and $\operatorname{Lie}\left(\widehat{S}_{\mathfrak{l}}\right)$ are related to (the $\sigma$-part of) $\operatorname{Tan}_{x}$ via appropriate scalar extension; therefore, the action of $M^{\times}$on $\operatorname{Lie}\left(\widehat{S}_{\mathfrak{p}}\right)$ can be transferred to $\operatorname{Lie}\left(\widehat{S}_{\mathfrak{l}}\right)$ via $\operatorname{Tan}_{x}$. We will see in Section 4 that $t_{\mathfrak{p}}$ (resp. $t_{\mathfrak{l}}$ ) is the exponential of the corresponding linear coordinate $\tau_{\mathfrak{p}}\left(\operatorname{resp} . \tau_{\mathfrak{l}}\right)$ of $\operatorname{Lie}\left(\widehat{S}_{\mathfrak{p}}\right)$ (resp. Lie $\left.\left(\widehat{S}_{\mathfrak{l}}\right)\right)$. From this, we conclude in Theorem 4.5 that $t_{\mathfrak{l}}$ also satisfies $t_{\mathfrak{l}} \circ \alpha=t_{\mathfrak{l}}^{\alpha^{\sigma(1-c)}}$ for $\alpha \in M^{\times}$, so, $\operatorname{End}_{F}^{0}\left(A_{\mathfrak{L} / \overline{\mathbb{F}}_{\mathfrak{l}}}\right)$ must be isomorphic to $M$. In Section 3 , we describe the canonical $O$-action on $\Omega_{S h / k}, \operatorname{Tan}_{x}$ and on $\operatorname{Lie}(\widehat{S})$, and in Section 4 , we study the $M$-action (extending the $O$-action) on the Serre-Tate coordinates (and its linear version).

Because of $x \in S h(k) \subset S h\left(\mathbb{C}_{p}\right)=S h\left(\mathbb{C}_{l}\right)$ (under our identification: $\mathbb{C}_{p}=\mathbb{C}_{l}$ ), the formal completion $\widehat{\mathcal{O}}_{S h, x}$ along the points $x \in S h\left(\mathbb{C}_{p}\right)=S h\left(\mathbb{C}_{l}\right)$ canonically contains $\mathcal{O}_{\widehat{S}_{p}}$ and $\mathcal{O}_{\widehat{S}_{l}}$. Thus in $\widehat{\mathcal{O}}_{S h, x}$ we can compare $\tau_{\mathfrak{p}}=\log _{p} \circ t_{\mathfrak{p}}$ and $\tau_{\mathfrak{l}}=\log _{l} \circ t_{\mathfrak{l}}$ for the $v$-adic $\operatorname{logarithm} \log _{v}$. Since they both satisfy $\tau_{\text {? }} \circ \alpha=\alpha^{\sigma(1-c)} \tau_{\text {? }}$, they are proportional; so, $\tau_{\mathfrak{l}}(A)=0$ and hence $t_{\mathfrak{l}}(A)=1$, which implies that the Tate module $T_{\mathfrak{l}} A$ of $A\left[\left[^{\infty}\right]\right.$ is also semi-simple under $D_{l}$. As is well known (cf. [O], 2.7 and Section 7 in this text), split ordinary primes for an abelian surface have Dirichlet density 1 for $k$ (replacing $k$ by its finite extension if necessary), and using the non-CM property of the $\mathfrak{p}$-adic Galois representation on the Tate module $T_{\mathfrak{p}} A$ (combined with Chebotarev density), we can find $\mathfrak{l}$ such that $M \nsubseteq \operatorname{End}_{F}^{0}\left(A_{\mathfrak{L} / \overline{\mathbb{F}}_{l}}\right)$, obtaining a contradiction (whose details will be given in Section [5). In the text, we fill details of this argument and generalize this to totally real fields $F$ with $[F: \mathbb{Q}] \geq 2$, to primes $\mathfrak{p}$ with residual degree $\geq 1$, and to abelian varieties $A$ which may not be ordinary at $\mathfrak{P}$ (i.e., only partially ordinary at $\mathfrak{P}$ ).

As a byproduct of our proof, we get (in Theorem 4.5) the proportionality of $\tau_{\mathfrak{p}}$ and $\tau_{\mathfrak{l}}$. Though it is irrelevant to the proof of the main theorem, in Section 6 we will explicitly compute the proportionality constant as the ratio of the square of the Katz $p$-adic and $l$-adic CM periods. In the above sketch of the proof, we heavily used the existence of many primes of $k$ at which the given abelian variety $A_{/ k}$ has good (partially) ordinary reduction. We give a brief account of the fact that such primes have density 1 among primes of (the Galois closure of) $k$ in Section 7 . 
As is clear from this sketch, there is much room for a generalization of our argument to abelian varieties with less multiplication. We hope to come back to the study of local indecomposability for general abelian varieties in the near future.

\section{Deformation Space of AN AVRM}

Pick a rational prime $p$. Let $F$ be a totally real field with integer ring $O$ in which $p$ is unramified. Write $d=[F: \mathbb{Q}]$. Let $(A, \lambda, \theta)_{/ k}$ be an abelian variety with real multiplication by $O$ (for short an AVRM by $O$ ) over a number field $k \subset \overline{\mathbb{Q}}$ with integer ring $\mathfrak{O}$ in which $p$ is unramified. Here $\theta: O \hookrightarrow \operatorname{End}\left(A_{/ k}\right)$ is a ring homomorphism sending the identity to the identity making $\operatorname{Lie}(A)$ a free $O \otimes_{\mathbb{Z}} k$ module of rank 1 , and $\lambda: A \rightarrow{ }^{t} A$ for the dual abelian scheme ${ }^{t} A=\operatorname{Pic}_{A / k}^{0}$ is an $O$-linear polarization of degree prime to $p$. Thus the $p$-adic Tate module $T_{p} A$ is free of rank 2 over $O_{p}=O \otimes_{\mathbb{Z}} \mathbb{Z}_{p}$, and $\operatorname{dim} A=[F: \mathbb{Q}]$. Let $\mathfrak{P}=\left\{x \in \mathfrak{O}:\left|i_{p}(x)\right|_{p}<1\right\}$ be the prime of $k$ induced by $i_{p}: \overline{\mathbb{Q}} \hookrightarrow \mathbb{C}_{p}$. Let $\overline{\mathbb{F}}_{p}=\overline{\mathbb{F}}_{\mathfrak{P}}$ be an algebraic closure of $\mathbb{F}_{\mathfrak{P}}=\mathfrak{O} / \mathfrak{P}$, and write $W=W_{p}$ for the ring of Witt vectors with coefficients in $\overline{\mathbb{F}}_{p}$. We identify $W$ with the subring of $\mathbb{C}_{p}$ which is the $p$-adic completion of the ring of integers in the maximal unramified extension of $\mathbb{Q}_{p}$ in $\overline{\mathbb{Q}}_{p} \subset \mathbb{C}_{p}$. Thus $i_{p}$ embeds $\mathfrak{O}$ in $W$, and we regard $\mathfrak{O}_{\mathfrak{P}} \subset W$. We put $\mathcal{W}=\mathcal{W}_{p}=i_{p}^{-1}\left(W_{p}\right) \subset \overline{\mathbb{Q}}$, which is a discrete valuation ring with residue field $\overline{\mathbb{F}}_{p}$ (and is a strict henselization of $\left.\mathbb{Z}_{(p)}=\mathbb{Q} \cap \mathbb{Z}_{p}\right)$.

Let $\mathfrak{O}_{\mathfrak{P}}$ be the $\mathfrak{P}$-adic completion of $\mathfrak{O}$, and suppose that $(A, \lambda, \theta)_{/ k}$ extends to the triple $(A, \lambda, \theta) / \mathfrak{O}_{\mathfrak{P}}$ for an abelian scheme $A_{/ \mathfrak{O}_{\mathfrak{P}}}$. Thus we can think of the special fiber $\left(A_{0}, \lambda_{0}, \theta_{0}\right)_{\mathbb{F}_{p}}=(A, \lambda, \theta) \times_{\mathfrak{O}_{\mathfrak{p}}} \overline{\mathbb{F}}_{p}$. Let $C L_{W}$ denote the category of complete local $W$-algebras with residue field $\overline{\mathbb{F}}_{p}$. We consider the fiber category $\mathcal{D}_{/ W}$ (over $C L_{W}$ ) of deformations of $\left(A_{0}, \lambda_{0}, \theta_{0}\right)_{/ \overline{\mathbb{F}}_{p}}$ over $R \in C L_{W}$. Objects of $\mathcal{D}_{/ W}$ are triples $\left(A^{\prime}, \lambda^{\prime}, \theta^{\prime}\right)_{/ R}$ satisfying $\iota_{A^{\prime}}:\left(A^{\prime}, \lambda^{\prime}, \theta^{\prime}\right) \times_{R} \overline{\mathbb{F}}_{p} \cong\left(A_{0}, \lambda_{0}, \theta_{0}\right)_{/ \overline{\mathbb{F}}_{p}} . \mathrm{A}$ morphism $\phi:\left(A^{\prime}, \lambda^{\prime}, \theta^{\prime}\right)_{/ R} \rightarrow\left(A^{\prime \prime}, \lambda^{\prime \prime}, \theta^{\prime \prime}\right)_{/ R}$ is a morphism of AVRM's $\phi: A^{\prime} \rightarrow A^{\prime \prime}$ such that

(1) the special fiber $\phi_{0}$ of $\phi$ satisfies $\iota_{A^{\prime \prime}} \circ \phi_{0} \circ \iota_{A^{\prime}}^{-1}=\mathrm{id}_{A_{0}}$ for the identity map $\operatorname{id}_{A_{0}}$ of $A_{0}$;

(2) $\lambda^{\prime}={ }^{t} \phi \circ \lambda^{\prime \prime} \circ \phi$

(3) $\phi \circ \theta^{\prime}(a)=\theta^{\prime \prime}(a) \circ \phi$ for all $a \in O$.

We suppose that

(NLL) the reduction $A_{0}:=A_{\mathfrak{P}}=A \otimes_{\mathfrak{O}_{\mathfrak{P}}} \overline{\mathbb{F}}_{p}$ has non-trivial $p$-torsion $\overline{\mathbb{F}}_{p}$-points.

So, we have a prime ideal $\mathfrak{p} \mid p$ of $O$ such that the $\mathfrak{p}$-adic Barsotti-Tate group $A\left[\mathfrak{p}^{\infty}\right]$ is ordinary, fitting into the following connected-étale exact sequence of Barsotti-Tate $O_{\mathfrak{p}}$-modules over $W$ :

$$
0 \rightarrow \mu_{p^{\infty}} \otimes_{\mathbb{Z}_{p}} O_{\mathfrak{p}} \rightarrow A\left[\mathfrak{p}^{\infty}\right] / W \rightarrow F_{\mathfrak{p}} / O_{\mathfrak{p}} \rightarrow 0 .
$$

Let $\Sigma^{\text {ord }}=\Sigma_{p}^{\text {ord }}$ be the set of primes $\mathfrak{p}$ in $O$ over $p$ such that $A\left[\mathfrak{p}^{\infty}\right] / W$ is not connected. Then we put $\Sigma^{l l}=\Sigma_{p}^{l l}=\left\{\mathfrak{p} \mid p: \mathfrak{p} \notin \Sigma^{\text {ord }}\right\}$. We may regard $\Sigma^{?}$ as a set of field embeddings $\sigma: F \hookrightarrow \overline{\mathbb{Q}}$ made up of $\sigma$ with $\left\{\alpha \in O:\left|i_{p}(\sigma(\alpha))\right|_{p}<1\right\}=\mathfrak{p} \in \Sigma^{\text {? }}$.

We now want to relate $\mathcal{D}_{/ W}$ with the following fiber category $D E F$ over $C L_{W}$ of quadruples $\left(A_{0}, D, \Lambda, \epsilon\right)_{/ R}$, where $D$ is a Barsotti-Tate $O$-module over $R, \Lambda: D \rightarrow$ ${ }^{t} D$ is an $O$-linear isomorphism of Barsotti-Tate groups into the Cartier dual ${ }^{t} D$ of $D$, and $\epsilon: D_{0} \cong A_{0}\left[p^{\infty}\right]$ is an $O$-linear isomorphism of Barsotti-Tate $O$-modules for 
the special fiber $D_{0}=D \otimes_{\operatorname{Spec}(W)} \operatorname{Spec}\left(\overline{\mathbb{F}}_{p}\right)$. We require $\epsilon$ to satisfy the following commutativity:

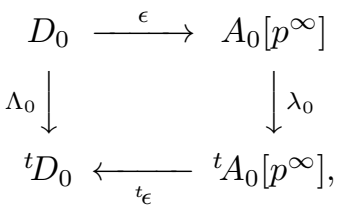

where $\Lambda_{0}$ is the special fiber of $\Lambda$. A morphism

$$
\phi:\left(A_{0}, D, \Lambda, \epsilon\right) \rightarrow\left(A_{0}^{\prime}, D^{\prime}, \Lambda^{\prime}, \epsilon^{\prime}\right)
$$

of $D E F$ is a pair of $O$-linear morphisms $\phi_{B T}: D \rightarrow D^{\prime}$ in $\operatorname{Hom}_{B T_{/ W}}\left(D, D^{\prime}\right)$ and $\phi_{\mathcal{D}}: A_{0} \rightarrow A_{0}^{\prime}$ in $\operatorname{Hom}_{G S C H_{/ \mathbb{F}_{p}}}\left(A_{0}, A_{0}^{\prime}\right)$ making the following two diagrams commutative:

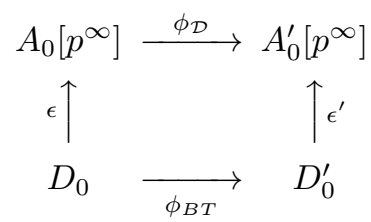

and

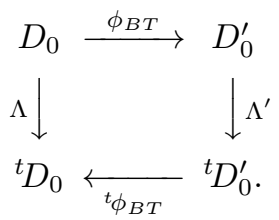

Here $G S C H$ (resp. $B T$ ) stands for the fiber category of group schemes (resp. Barsotti-Tate groups) over $C L_{W}$. We have a natural functor: $\mathcal{D} / W \rightarrow D E F$ given by

$$
A \mapsto\left(A_{0}=A \otimes_{W} \overline{\mathbb{F}}_{p}, A\left[p^{\infty}\right],\left.\lambda\right|_{A\left[p^{\infty}\right]}, \iota\right)
$$

for the canonical isomorphism $\iota: A\left[p^{\infty}\right] \otimes_{W} \overline{\mathbb{F}}_{p} \cong A_{0}\left[p^{\infty}\right]$.

Theorem 1.1 (Serre-Tate). The above functor: $\mathcal{D}_{/ W} \rightarrow D E F$ is a canonical equivalence of categories.

A proof of this is given in $[\mathrm{K}$, though the input of $\theta$ and $\lambda$ is not there (see [K1], Lemma 1.11.6 or [Ra], $\S 1.7$ on how to modify the argument incorporating endomorphisms and a polarization).

We can split $D E F$ into the fibered product of the following fiber categories: $D E F^{\text {ord }}$ and $D E F^{l l}$ over $C L_{W}$. Here "ord" stands for ordinary Barsotti-Tate groups and " $l l$ " stands for local-local Barsotti-Tate groups. The objects of $D E F$ ? are given by $\left(A_{0}, D^{?}, \Lambda^{?}, \epsilon^{?}\right) / R$ such that $D^{?}$ is a Barsotti-Tate $O$-module deforming $A_{0}\left[p^{\infty}\right]^{?}=\prod_{\mathfrak{p} \in \Sigma^{?}} A_{0}\left[\mathfrak{p}^{\infty}\right]$ for $?=\operatorname{ord}, l l$ and $\Lambda^{?}: D^{?} \cong{ }^{t} D^{?}$ is an $O$-linear isomorphism and $\epsilon^{?}: D_{0}^{?} \cong A_{0}\left[p^{\infty}\right]^{\text {? }}$. Thus $D^{l l}$ is local-local (i.e., $D^{l l}$ and ${ }^{t} D^{l l}$ are both connected). The quadruple $\left(A_{0}, D^{?}, \Lambda^{?}, \epsilon^{?}\right)$ is supposed to satisfy the same compatibility of diagrams defining $D E F$. Since by $O$-linearity we have a canonical decomposition $D=D^{\text {ord }} \times D^{l l}$ for an object $\left(A_{0}, D, \Lambda, \epsilon\right) / R$ of $D E F$ with the maximal ordinary Barsotti-Tate $O$-module $D^{\text {ord }}$ in $D$ and the local-local complement 
$D^{l l}$ of $D^{\text {ord }}$ in $D$ so that $D=D^{\text {ord }} \times D^{l l}$, we get a decomposition

$$
\begin{aligned}
D E F & \ni\left(A_{0}, D, \Lambda, \epsilon\right) \mapsto\left\{\left(A_{0}, D^{\text {ord }},\left.\Lambda\right|_{D^{\text {ord }}},\left.\epsilon\right|_{D^{\text {ord }}}\right),\left(A_{0}, D^{l l},\left.\Lambda\right|_{D^{l l}}, \epsilon^{l l}\right)\right\} \\
& \in D E F^{\text {ord }} \times D E F^{l l}
\end{aligned}
$$

which induces the equivalence between $D E F$ and $D E F^{o r d} \times D E F^{l l}$.

We consider the deformation functor $\widehat{D}: C L / W \rightarrow S E T S$ given by

$$
\widehat{D}(R)=\left\{(A, \lambda, \theta)_{/ R} \mid(A, \lambda, \theta)_{/ R} \in \mathcal{D}\right\} / \cong .
$$

To describe the deformation space representing $\widehat{D}$, we write $O_{\Xi}=\prod_{\mathfrak{l} \in \Xi} O_{\mathfrak{l}}$ for the $\Xi$-adic completion of $O$ for any finite set of prime ideals $\Xi$ of $O$. Then we put $F_{\Xi}=O_{\Xi} \otimes_{O} F$ and $O_{(\Xi)}=F \cap O_{\Xi}$ in $F_{\Xi}$.

Proposition 1.2. Let the notation be as above. Suppose that $p$ is unramified in $F / \mathbb{Q}$ and in $k / \mathbb{Q}$. Then the deformation functor $\widehat{D}$ is representable by the smooth formal scheme $\widehat{S}_{/ W}$ which is a product of two smooth formal schemes: $\widehat{S}^{\text {ord }}$ isomorphic to $\widehat{\mathbb{G}}_{m} \otimes_{\mathbb{Z}_{p}} O_{\Sigma^{\text {ord }}}=\prod_{\mathfrak{p} \in \Sigma^{\text {ord }}} \widehat{\mathbb{G}}_{m} \otimes_{\mathbb{Z}_{p}} O_{\mathfrak{p}}$ and $\widehat{S}^{l l}$, where $\widehat{S}^{l l} \cong$ $\operatorname{Spf}\left(W\left[\left[T_{1}, \ldots, T_{m}\right]\right]\right)$ (non-canonically) for $m=\operatorname{rank}_{\mathbb{Z}_{p}} O_{\Sigma^{l l}}$, and each factor $\widehat{S}^{\text {? }}$ represents the formal stack $D E F^{\text {? }}$.

Proof. As is well known, the formal stack $D E F$ over $W$ is represented by a formal scheme $\widehat{S}$ smooth over $W$ of relative dimension equal to $\operatorname{rank}_{\mathbb{Z}_{p}} O_{p}$. Indeed, adding an $O$-linear prime-to- $p$ adelic level structure $\eta^{(p)}:\left(\widehat{O}^{(p)}\right)^{2} \cong T^{(p)} A=$ $\lim _{p \nmid N} A_{0}[N]\left(\overline{\mathbb{F}}_{p}\right)$ to $\left(A_{0}, \lambda_{0}, \theta_{0}\right)$, the quadruple $\left(A_{0}, \lambda_{0}, \theta_{0}, \eta^{(p)}\right)$ (called a test object) gives rise to an $\overline{\mathbb{F}}_{p}$-point $x_{0}$ of the Hilbert modular Shimura variety $S h_{/ W}^{(p)}=$ $S h_{/ \mathbb{Z}_{(p)}}^{(p)} \times W$ of prime-to- $p$ level. In Section 2 we recall the definition of the Shimura variety. Then $\widehat{S}_{/ W}$ can be identified with the formal completion of $S h_{/ W}^{(p)}$ along $x_{0}$ (and the Shimura variety is smooth over $\mathbb{Z}_{(p)}$ of relative dimension equal to $\left.\operatorname{rank}_{\mathbb{Z}_{p}} O_{p}=[F: \mathbb{Q}]\right)$. For the part $D E F^{\text {ord }}$, by the theory of Serre-Tate, once we identify $A_{0}\left[p^{\infty}\right]^{\text {ord }}$ with $\left(\mu_{p^{\infty}} \otimes_{\mathbb{Z}_{p}} O_{\Sigma_{\text {ord }}}\right) \times\left(F_{\Sigma^{\text {ord }}} / O_{\Sigma_{\text {ord }}}\right)$ by a level $p$-structure

$$
\eta_{p}:\left(\mu_{p \infty} \otimes_{\mathbb{Z}_{p}} O_{\Sigma_{\text {ord }}}\right) \times\left(F_{\Sigma_{\text {ord }}} / O_{\Sigma_{\text {ord }}}\right) \cong A_{0}\left[p^{\infty}\right]^{\text {ord }},
$$

the space $\widehat{S}^{\text {ord }}$ is isomorphic to $\widehat{\mathbb{G}}_{m} \otimes_{\mathbb{Z}_{p}} O_{\Sigma \text { ord }}$ whose origin corresponds to the unique canonical lift of $A_{0}\left[p^{\infty}\right]^{\text {ord }}$ with complex multiplication (which may be different from the starting $A\left[p^{\infty}\right]^{\text {ord }}$ ). Since $D E F \approx D E F^{\text {ord }} \times D E F^{l l}$ (the equivalence of categories), we have a product decomposition $\widehat{S}=\widehat{S}^{\text {ord }} \times \widehat{S}^{l l}$. Since $\widehat{S}$ and $\widehat{S}^{\text {ord }}$ are formally smooth, $\widehat{S}^{l l}$ must be formally smooth. Then by dimension computation, we have $\operatorname{dim}_{W} \widehat{S}^{l l}=m$ as above.

We have $\widehat{S}_{p}^{\text {ord }} \cong \widehat{\mathbb{G}}_{m} \otimes_{\mathbb{Z}_{p}} O_{\Sigma^{\text {ord }}}=\prod_{\mathfrak{p} \in \Sigma^{\text {ord }}} \widehat{S}_{\mathfrak{p}}^{\text {ord }}$ for $\widehat{S}_{\mathfrak{p}}^{\text {ord }}=\widehat{\mathbb{G}}_{m} \otimes_{\mathbb{Z}_{p}} O_{\mathfrak{p}}$. Thus $\widehat{S}_{\mathfrak{p}}^{\text {ord }}$ is the $O_{\mathfrak{p}}$-factor of the formal completion of $\mathbb{G}_{m} \otimes_{\mathbb{Z}} O=\operatorname{Spec}\left(W\left[t^{\xi}\right]_{\xi \in O}\right)$ along the origin corresponding to the maximal ideal $(p, t-1)$. Here we used the fact that for the cocharacter group $L$ of a split torus $T_{/ W}$, we have a canonical isomorphism: $T \cong \operatorname{Spec}\left(W\left[t^{l}\right]_{l \in L}\right)=\mathbb{G}_{m} \otimes_{\mathbb{Z}} L$ for the group algebra $W[L] \cong W\left[t^{l}\right]_{l \in L}$ with the canonical variable $t$ of $\mathbb{G}_{m}$. Since $\widehat{\mathbb{G}}_{m} \otimes_{\mathbb{Z}} O=\widehat{\mathbb{G}}_{m} \otimes_{\mathbb{Z}} O_{p}\left(O_{p}=\lim _{n} O / p^{n} O\right)$, $\widehat{\mathbb{G}}_{m} \otimes_{\mathbb{Z}_{p}} O_{\mathfrak{p}}$ has variable $t_{\mathfrak{p}}=t \otimes 1_{\mathfrak{p}}$ for the idempotent $1_{\mathfrak{p}}$ of $O_{\mathfrak{p}}$ in $O_{p}=\prod_{\mathfrak{p}^{\prime} \mid p} O_{\mathfrak{p}^{\prime}}$. We call $t_{\mathfrak{p}}$ the Serre-Tate (multiplicative) coordinate of $\widehat{S}_{\mathfrak{p}}^{\text {ord }}$. 


\section{Hilbert modular Shimura variety}

Here is a more detailed definition of AVRM by $O$. Write $O^{*}=\operatorname{Hom}_{\mathbb{Z}}(O, \mathbb{Z})$ (the $\mathbb{Z}$-linear dual), which can be identified with the different inverse of $F_{/ \mathbb{Q}}$ by the trace pairing. A triple $(A, \lambda, \theta) / S$ with an abelian scheme $A$ over a scheme $S$ with real multiplication is called an AVRM by $O$ if it satisfies the following four conditions:

(rm1) $\theta=\theta_{A}: O \hookrightarrow \operatorname{End}\left(A_{/ S}\right)$ is an embedding of algebras taking the identity to the identity.

(rm2) $\lambda$ is an $O$-linear symmetric isogeny $\lambda: A \rightarrow{ }^{t} A$ induced by an ample line bundle fiber-by-fiber geometrically (see GIT], 6.2). Identifying ${ }^{t} A$ with $A \otimes_{O} \mathfrak{c}$ for a fractional ideal $\mathfrak{c}$ of $F, \lambda$ is called a $\mathfrak{c}$-polarization of $A$. Here $\lambda$ is called symmetric if $\lambda={ }^{t} \lambda$.

(rm3) The image of $\theta_{A}$ is stable under the Rosati involution on the endomorphism algebra $\operatorname{End}^{0}(A): \alpha \mapsto \alpha^{*}=\lambda^{-1} \circ{ }^{t} \alpha \circ \lambda$.

(rm4) As $O \otimes_{\mathbb{Z}} \mathcal{O}_{S}$-modules, we have an isomorphism: $\operatorname{Lie}(A) \cong O \otimes_{\mathbb{Z}} \mathcal{O}_{S}(\Leftrightarrow$ $\pi_{*}\left(\Omega_{A / S}\right) \cong O^{*} \otimes_{\mathbb{Z}} \mathcal{O}_{S}$ for $\left.\pi: A \rightarrow S\right)$ locally under the Zariski topology of $S$, where the sheaf $\operatorname{Lie}(A)$ of Lie algebras of $A$ (i.e., the pull-back of the tangent bundle over $A_{/ S}$ by the 0 -section) is an $O$-module by the action induced from $\theta$.

An AVRM $A$ defined over a field $\kappa$ (potentially) has complex multiplication (or has CM) if the algebra $\operatorname{End}_{F}^{0}\left(A \times_{\kappa} \bar{\kappa}\right)=\operatorname{End}_{O}\left(A \times_{\kappa} \bar{\kappa}\right) \otimes_{O} \mathbb{Q}$ (of $F$-linear endomorphisms of $A \times{ }_{\kappa} \bar{\kappa}$ defined over $\bar{\kappa}$ ) for an algebraic closure $\bar{\kappa}$ of $\kappa$ is not equal to $F$ (and if $\kappa \subset \overline{\mathbb{Q}}$ and $A$ has $\mathrm{CM}, \operatorname{End}_{F}^{0}\left(A \times_{\kappa} \bar{\kappa}\right)$ is a quadratic extension of $F$ ). An AVRM $A$ over an integral domain $\mathfrak{r}$ has CM if $A \times_{\mathfrak{r}} \kappa$ has CM for the quotient field $\kappa$ of $\mathfrak{r}$. An AVRM $A / \mathfrak{r}$ is said to be $n o n-C M$ if $\operatorname{End}_{F}^{0}\left(A \times_{\mathfrak{r}} \bar{\kappa}\right)=F$.

In the rest of the paper, we denote by $\Xi$ a finite set of rational primes unramified in $F$ containing the fixed prime $p$, and we put $\mathbb{Z}_{(\Xi)}=\bigcap_{l \in \Xi}\left(\mathbb{Q} \cap \mathbb{Z}_{l}\right) \subset \mathbb{Q}$ (the localization at $\Xi$ of $\mathbb{Z})$. We write $O_{(\Xi)}$ for the ring $O \otimes_{\mathbb{Z}} \mathbb{Z}_{(\Xi)} \subset F$ and let $O_{(\Xi)+}^{\times}$ be the group of totally positive units in $O_{(\Xi)}$. Let $\mathcal{A}^{(\Xi)}$ be the fiber category over $\mathbb{Z}_{(\Xi)}$-schemes made of abelian schemes with real multiplication by $O$ up to primeto- $\Xi$ isogenies. Thus the objects of $\mathcal{A}^{(\Xi)}$ are triples $\left(A, \bar{\lambda}, \theta_{A}\right)_{/ S}$ over a $\mathbb{Z}_{(\Xi) \text {-scheme }}$ $S$, and $\bar{\lambda}$ is the set $\left\{a \lambda:=\lambda \circ \theta_{A}(a)\right\}_{a \in O_{(\Xi)+}^{\times}}$for a polarization $\lambda$ with a polarization ideal prime to $\Xi$. The morphisms $\left(A, \bar{\lambda}, \theta_{A}\right)_{/ S} \rightarrow\left(A^{\prime}, \bar{\lambda}^{\prime}, \theta_{A}^{\prime}\right) / S$ of $\mathcal{A}^{(\Xi)}$ are given by the elements $\phi: A \rightarrow A^{\prime}$ in $\operatorname{Hom}_{G S C H_{/ \mathbb{Z}}(\Xi)}\left(A, A^{\prime}\right) \otimes_{\mathbb{Z}} \mathbb{Z}_{(\Xi)}$ compatible with the data $\left(\bar{\lambda}, \theta_{A}\right)$ and $\left(\bar{\lambda}^{\prime}, \theta_{A^{\prime}}\right)$. Here $G S C H_{/ R}$ stands for the category of group schemes over a ring $R$. We consider the Hilbert modular Shimura variety $S h_{/ \mathbb{Z}_{(\Xi)}}^{(\Xi)}$ which is known to represent the stack made out of $\left(A, \bar{\lambda}, \theta_{A}, \eta^{(\Xi)}\right)$ for a prime-to- $\Xi O$-linear level structure $\eta^{(\Xi)}:\left(F_{\mathbb{A}}^{(\Xi, \infty)}\right)^{2} \cong T^{(\Xi)} A \otimes_{\mathbb{Z}} \mathbb{Z}_{(\Xi)}$ sending the determinant pairing $(a, b) \mapsto \operatorname{det}(a, b)$ for $a, b \in\left(F_{\mathbb{A}}^{(\Xi, \infty)}\right)^{2}$ to the pairing on $T^{(\Xi)} A$ induced by the polarization up to similitude factors in $\left(F_{\mathbb{A}}^{(\Xi, \infty)}\right)^{\times}$, where $T^{(\Xi)} A$ is the prime-to- $\Xi$ Tate module of $A, F_{\mathbb{A}}$ is the adele ring of $F$ and $F_{\mathbb{A}}^{(\Xi, \infty)}$ is its prime-to- $\Xi$ part given by $\left\{x \in F_{\mathbb{A}} \mid x_{l}=x_{\infty}=0\right.$ for all $\left.l \in \Xi\right\}$. Strictly speaking, to get $S h_{\mathbb{Z}_{(l)}}^{(\Xi)}(l \in \Xi)$ for $\Xi^{(l)}:=\Xi \backslash\{l\}$, we need to actually identify the $\Xi^{(l)}$-part of the Tate module $T_{\Xi^{(l)}} A$ with $O_{\Xi(l)}^{2}$ (without specifying an isomorphism; see [H06, $\S 1$ for more details). 
Then the schemes $\left\{S h_{/ \mathbb{Z}_{(l)}}\right\}_{l \in \Xi}$ glue into $S h_{/ \mathbb{Z}_{(\Xi)}}^{(\Xi)}$. The pro-scheme $S h^{(\Xi)}$ is smooth over $\mathbb{Z}_{(\Xi)}$ (cf. $[\mathrm{PAF}$, Section 4.2 ).

Let $G=\operatorname{Res}_{F / \mathbb{Q}} G L(2)$ with center $Z_{/ \mathbb{Q}}$. Under the action $\eta^{(\Xi)} \mapsto \eta^{(\Xi)} \circ g$, each $g \in G\left(\mathbb{A}^{(\Xi), \infty}\right)$ acts on $S h^{(\Xi)}$ as an automorphism. Let $\mathcal{W}_{\Xi}=\bigcap_{l \in \Xi} \mathcal{W}_{l}$ (inside $\overline{\mathbb{Q}}$ ), which is a henselian semi-local Dedekind domain with localization at each maximal ideal $\mathfrak{m}_{l}$ of residual characteristic $l$ equal to $\mathcal{W}_{l}$. We fix a non-CM test object $\left(A, \bar{\lambda}, \theta, \eta^{(\Xi)}\right) / \mathcal{W}_{\Xi}$ satisfying (NLL). Let $x \in S h^{(\Xi)}\left(\mathcal{W}_{\Xi}\right)$ be the point representing this test object.

Suppose $p \in \Xi$. By a theorem of Tate (cf. ABV], Appendix I), the reduction $\left(A_{0}, \bar{\lambda}_{0}, \theta_{0}, \eta_{0}^{(\Xi)}\right)_{/ \overline{\mathbb{F}}_{p}}=\left(A, \lambda, \theta, \eta^{(\Xi)}\right) / \mathcal{W}_{\Xi} \times \mathcal{W}_{\Xi} \overline{\mathbb{F}}_{p}$ has complex multiplication by a field $M=\operatorname{End}_{F}^{0}\left(A_{0 / \overline{\mathbb{F}}_{p}}\right)$ (an $F$-linear endomorphism algebra). Regarding $M \subset$ $\overline{\mathbb{Q}}$ as an $F$-algebra, we write $\widetilde{\theta}_{\mathfrak{P}}: M \cong \operatorname{End}_{F}^{0}\left(A_{0 / \overline{\mathbb{F}}_{p}}\right)$ for the identification (so, $\left.\left.\widetilde{\theta}_{\mathfrak{P}}\right|_{F}=\theta_{0}\right)$. Let $R=\operatorname{End}_{O}\left(A_{0 / \overline{\mathbb{F}}_{p}}\right)$, which is an order of $M$. The quadruple $\left(A_{0}, \bar{\lambda}_{0}, \theta_{0}, \eta_{0}^{(\Xi)}\right) / \overline{\mathbb{F}}$ gives rise to a point $x_{0} \in S h^{(\Xi)}\left(\overline{\mathbb{F}}_{p}\right)$. Let $V$ be the geometrically irreducible component of $S h_{/ \mathcal{W} \Xi}^{(\Xi)}$ containing $x$, so $V_{/ \overline{\mathbb{F}}_{p}}=V \times_{\mathcal{W} \Xi} \overline{\mathbb{F}}_{p}$ is geometrically irreducible and contains $x_{0}$. By the smoothness of $S h^{(\Xi)}$ over $\mathbb{Z}_{(\Xi)}$ and the existence of the smooth projective toroidal compactification of $S h^{(\Xi)}, V_{/ \overline{\mathbb{F}}_{p}}$ is geometrically connected.

By the global reciprocity law of Shimura (cf. [Sh], II), we know

Theorem 2.1. Let the notation and the assumption be as above. Suppose $p \in \Xi$, which is unramified in $F / \mathbb{Q}$. Let $\kappa=\overline{\mathbb{F}}_{p}$ or $\overline{\mathbb{Q}}$. For the geometrically connected component $V_{/ \kappa} \subset S h_{/ \mathbb{Z}_{(\Xi)}}^{(\Xi)} \times_{\mathbb{Z}_{(\Xi)}} \kappa$, the scheme automorphism group $\operatorname{Aut}\left(V_{/ \kappa}\right) \subset$ $G\left(\mathbb{A}^{(\Xi), \infty}\right)$ is given by the semi-direct product of the field automorphism group $\operatorname{Aut}\left(F_{/ \mathbb{Q}}\right)$ and

$$
\overline{\mathcal{E}}^{(\Xi)}=\frac{\left\{g \in G\left(\mathbb{A}^{(\Xi)}\right) \mid \operatorname{det}(g) \in \overline{O_{(\Xi)+}^{\times} F_{\infty+}^{\times}}\right\}}{\overline{Z\left(\mathbb{Z}_{(\Xi)}\right) G(\mathbb{R})_{+}}}=\frac{\left\{g \in G\left(\mathbb{A}^{(\Xi), \infty}\right) \mid \operatorname{det}(g) \in \overline{O_{(\Xi)+}^{\times}}\right\}}{\overline{Z\left(\mathbb{Z}_{(\Xi)}\right)}},
$$

where $G(\mathbb{R})_{+}$is the identity connected component of $G(\mathbb{R})$ and " $\cdots$ " indicates closure under adelic topology. Here $\operatorname{Aut}\left(F_{/ \mathbb{Q}}\right)$ acts on $\overline{\mathcal{E}}^{(\Xi)}$ through its action on $G(\mathbb{A})$. For the above point $x_{0} \in S h^{(\Xi)}\left(\overline{\mathbb{F}}_{p}\right)$, the stabilizer of $x_{0}$ in $\overline{\mathcal{E}}^{(\Xi)}$ is the image of the morphism $\widehat{\rho}: R_{(\Xi)}^{\times} \rightarrow G\left(\mathbb{A}^{(\Xi), \infty}\right)$ given by $\widetilde{\theta}_{\mathfrak{P}}(\alpha) \circ \eta_{0}^{(\Xi)}=\eta_{0}^{(\Xi)} \circ \widehat{\rho}(\alpha)$ for $\alpha \in R_{(\Xi)}^{\times}=\left(R \otimes_{\mathbb{Z}} \mathbb{Z}_{(\Xi)}\right)^{\times} \subset M^{\times}$.

By this theorem, the stabilizer $\mathcal{T}$ in $\overline{\mathcal{E}}=\operatorname{Aut}\left(V_{/ \overline{\mathbb{F}}_{p}}\right)$ of $x_{0}$ is the image of $R_{(\Xi)}^{\times}$for the order $R$ in the integer ring $\mathfrak{o}$ of $M$ under the morphism $\widehat{\rho}: T_{M}=\operatorname{Res}_{R / \mathbb{Z}} \mathbb{G}_{m} \rightarrow G$ of group schemes defined over $\mathbb{A}^{(\Xi), \infty}$ (so, it is a conjugate of an anisotropic torus in $G$ under an element in $G\left(\mathbb{A}^{(\Xi), \infty)}\right)$. It would appear later that we do not use this theorem, but it will be implicitly used to assure that our action of $T_{M}\left(\mathbb{Z}_{(\Xi)}\right)$ preserves each geometrically irreducible component of $S h^{(\Xi)}$ as its action factors through $\overline{\mathcal{E}}^{(\Xi)}$.

Proof. The first assertion is proven by T. Miyake in $[\mathrm{M}]$ for $\kappa=\overline{\mathbb{Q}}$ (following the method of Shimura in Sh, II) via the well-known identification of the automorphism group of a bounded symmetric domain with the corresponding Lie 
group (e.g., DLS], Chapter IV); in our Hilbert modular case, $\operatorname{Aut}\left(\mathfrak{H}^{I}\right)(\mathfrak{H}=\{z \in$ $\mathbb{C} \mid \operatorname{Im}(z)>0\})$ has an identity connected component isomorphic to $P S L_{2}(\mathbb{R})^{I}$ for $I=\operatorname{Hom}_{\text {field }}(F, \overline{\mathbb{Q}})$. See $[\mathrm{PAF}]$, Theorem 4.14 for another algebraic proof for $\kappa=\overline{\mathbb{Q}}$, and the case where $\kappa=\overline{\mathbb{F}}_{p}$ is treated in [H06]. If $g \in \overline{\mathcal{E}}^{(\Xi)}$ stabilizes $x_{0}$, by universality, we have a prime-to- $\Xi F$-linear endo-isogeny

$$
\alpha:\left(A_{0}, \bar{\lambda}_{0}, \theta_{0}, \eta_{0}^{(\Xi)}\right)_{/ \overline{\mathbb{F}}_{p}} \cong\left(A_{0}, \bar{\lambda}_{0}, \theta_{0}, \eta_{0}^{(\Xi)} \circ g\right)_{/ \overline{\mathbb{F}}_{p}} .
$$

In other words, $\alpha$ can be regarded as an element of $R_{(\Xi)}^{\times}$. Since $\widetilde{\theta}_{\mathfrak{P}}(\alpha) \circ \eta^{(\Xi)}=$ $\eta^{(\Xi)} \circ \widehat{\rho}(\alpha)$, the matrix $\widehat{\rho}(\alpha)$ coincides with $g$ in the quotient $\overline{\mathcal{E}}^{(\Xi)}$.

Lemma 2.2. Suppose that the AVRM $\left(A_{0}, \bar{\lambda}\right)_{/ \overline{\mathbb{F}}_{p}}$ is defined over a finite field $\mathbb{F}_{q} \subset$ $\overline{\mathbb{F}}_{p}$ and $\mathfrak{p} \in \Sigma^{\text {ord }}$. Then the algebra $M=\operatorname{End}_{F}^{0}\left(A_{0 / \overline{\mathbb{F}}_{p}}\right)$ is a $C M$ quadratic extension $M$ over $F$ generated by the Frobenius endomorphism over $\mathbb{F}_{q}$. In particular, the prime $\mathfrak{p}$ in $F$ splits into $\mathcal{P} \overline{\mathcal{P}}$ in $R$ for primes $\mathcal{P} \neq \overline{\mathcal{P}}$. of $M$

By this lemma, we have $R_{\mathfrak{p}}=\mathfrak{o}_{\mathfrak{p}}=R_{\mathcal{P}} \times R_{\overline{\mathcal{P}}} \cong O_{\mathfrak{p}} \times O_{\mathfrak{p}}$ for the integer ring $\mathfrak{o}$

Proof. By definition, $M_{\mathfrak{p}}$ is isomorphic to the $F$-linear endomorphism algebra $\operatorname{End}_{F}^{0}\left(A_{0}\left[\mathfrak{p}^{\infty}\right]_{/ \mathbb{F}_{q}}\right)$, which is therefore a semi-simple quadratic extension of $F_{\mathfrak{p}}$. Thus $M$ is commutative, and the embedding restricted to $M \subset M_{\mathfrak{p}}$ factors through $\operatorname{End}_{F}^{0}\left(A_{0 / \mathbb{F}_{q}}\right)$ and its image contains the $q$-th power Frobenius map $\phi$. Since $F[\phi] / F$ is a CM field because of the positivity and non-triviality of the Rosati involution $c$ of $\lambda_{0}$, we must have $M=F[\phi]$, which is a CM quadratic extension of $F$.

Note that $\phi$ and its dual $\phi^{c}=\lambda^{-1} \circ{ }^{t} \phi \circ \lambda$ satisfy $\phi \phi^{c}=\phi^{c} \phi=q$. Since $\phi$ is invertible on $A_{0}\left(\overline{\mathbb{F}}_{p}\right) \supset A_{0}\left[\mathfrak{p}^{\infty}\right] \neq 0, a=\phi+\phi^{c} \in O$ is prime to $\mathfrak{p}$. Thus the minimal polynomial $X^{2}-a X+q$ of $\phi$ over $F$ satisfies $X^{2}-a X+q \equiv X(X-a) \bmod \mathfrak{p}$ with $(a \bmod \mathfrak{p}) \neq 0 ;$ so, $\mathfrak{p}$ must split in $R \supset O[\phi]$.

We often choose $\mathcal{P}$ so that $\mathcal{P}$ contains $\phi$; therefore, $A_{0}[\mathcal{P}]$ is connected.

\section{EIGENDIFFERENTIALS}

We keep the notation introduced in Theorem 2.1 in the rest of the paper. For an open compact subgroup $K$ of $G\left(\mathbb{A}^{(\Xi), \infty)}\right.$, we write $V_{K}$ for the image of $V$ under $V \subset S h^{(\Xi)} \rightarrow S h_{K}^{(\Xi)}$ for the geometric quotient $S h_{K}^{(\Xi)}:=S h^{(\Xi)} / K$ (thus $V$ is the pro-variety $V=\lim _{K} V_{K}$ in the sense of Grothendieck [EGA], IV.8 or [EAI], §5.1.4). We take $K$ sufficiently small so that $K$ is neat; i.e.,

$$
\Gamma_{g K g^{-1}}=\left\{\alpha \in g K g^{-1} \cap G(\mathbb{Q}) \mid \operatorname{det}(\alpha) \text { is totally positive }\right\}
$$

does not have torsion modulo its center (this holds true if $K$ is inside the principal congruence subgroup of level $N \geq 3$ ). Under neatness, $V_{K}$ is smooth over $\mathcal{W}_{\Xi}$.

Let $\pi:\left(\mathbf{A}, \theta_{\mathbf{A}}\right) \rightarrow V_{K}$ be the universal AVRM over $V_{K}$. Then $\underline{\omega}=\pi_{*} \Omega_{\mathbf{A} / V_{K}}$ has an $O$-action via $\theta_{\mathbf{A}}$ and by (rm4) is locally free of rank 1 over $O \otimes_{\mathbb{Z}} \mathcal{O}_{V_{K}}$ (which is a module over $\left.O \otimes_{\mathbb{Z}} \mathcal{W}_{\Xi}\right)$. Since $O \otimes_{\mathbb{Z}} \mathcal{W}_{\Xi}=\mathcal{W}_{\Xi}^{I}$ by sending $a \otimes b$ to $\varphi(a) b$ for $\varphi \in I=\operatorname{Hom}_{\text {field }}(F, \overline{\mathbb{Q}}$ ) (as primes in $\Xi$ are unramified in $O$ ), we can accordingly split $\underline{\omega}=\bigoplus_{\varphi \in I} \underline{\omega}^{\varphi}$ for a line bundle $\underline{\omega}^{\varphi}$ on which the $O$-action factors through $\varphi$. By the Kodaira-Spencer map (see $[\mathrm{K}], \S 3.6), \Omega_{V_{K} / \mathcal{W}_{\Xi}}$ is canonically isomorphic to $\bigoplus_{\varphi} \underline{\omega}^{2 \varphi}$ for $\underline{\omega}^{2 \varphi}=\underline{\omega}^{\varphi} \otimes \mathcal{O}_{V_{K}} \underline{\omega}^{\varphi}$ (see [K1], 1.0.21). Thus the cotangent space 
$\Omega_{V_{K} / \mathcal{W}}$ has a canonical $O_{(\Xi)}^{\times}$-action. A section $T$ of the structure sheaf of a Zariski (resp. complex analytic, $p$-adic rigid analytic) neighborhood $D_{x}$ (of $x$ ) is called "belonging to $\varphi$ " if $d T \in H^{0}\left(D_{x},\left(\underline{\omega}^{2 \varphi}\right)^{*}\right)$ for the line bundle $\left(\underline{\omega}^{2 \varphi}\right)^{*}$ corresponding to the algebraic bundle $\underline{\omega}^{2 \varphi}$ (by GAGA theorems; see Example 2.4.6 in [C]). Here we use $*=a$ an in the complex analytic case and in the rigid analytic case (and we place nothing in the algebraic case; if we need to specify the place $v$, we write $*=v$-an). If $T$ is a part of the coordinate system belonging to $\varphi$ (at $v \in \Xi \cup\{\infty\}$ ), we call $T$ a $\varphi$-coordinate (at $v$ ).

Recall $M=\operatorname{End}_{F}^{0}\left(A_{0}\right) \supset \operatorname{End}_{O}\left(A_{0}\right)=R$. The field $M$ is a CM quadratic extension of $F$, and let $T_{M}=\operatorname{Res}_{R / \mathbb{Z}} \mathbb{G}_{m}$. Recall the morphism $\widehat{\rho}: T_{M} \rightarrow G(\mathbb{A}(\Xi), \infty)$ of algebraic groups over $\mathbb{A}^{(\Xi), \infty}$ given by $\widetilde{\theta}_{\mathfrak{P}}(\alpha) \circ \eta_{0}^{(\Xi)}=\eta_{0}^{(\Xi)} \circ \widehat{\rho}(\alpha)$. The point $x_{0} \in$ $V\left(\overline{\mathbb{F}}_{p}\right)$ is a fixed point of $\widehat{\rho}\left(T_{M}\left(\mathbb{Z}_{(\Xi)}\right)\right)$, and let $U$ be a Zariski open neighborhood of $x_{0}$. Suppose that we have a non-constant $f \in \overline{\mathbb{F}}_{p}(V)$ vanishing at $x_{0}$ and a $p$-adically open subgroup $\mathcal{T}$ of $T_{M}\left(\mathbb{Z}_{(\Xi)}\right)$ such that $f \circ \widehat{\rho}(\alpha)=\lambda_{\alpha} f$ with a constant $\lambda_{\alpha} \in \overline{\mathbb{F}}_{p}$ for all $\alpha \in \mathcal{T}$. Let $X^{\circ}=\{u \in U \mid f(u)=0\}$ and $X$ be the Zariski closure of $X^{\circ}$. Since $f$ is non-constant, $\operatorname{dim} X=d-1$. Then, by definition, $X$ is stable under the action of the image $\mathcal{T} \subset \overline{\mathcal{E}}^{(\Xi)}$ of $\widehat{\rho}\left(T_{M}\left(\mathbb{Z}_{(\Xi)}\right)\right)$. Supposing that $x_{0}$ is in the ordinary locus, by [H10], Proposition 3.8, $X=V$ if $\operatorname{dim} X=d-1>0$. This is impossible, so such a non-trivial $f$ does not exist.

Suppose that we have a flat integral covering space $\widetilde{U} / U$ whose image contains $x_{0}$ on which the action of $\mathcal{T}$ extends. If $f \circ \widehat{\rho}(\alpha)=\lambda_{\alpha} f$ with a constant $\lambda_{\alpha} \in \overline{\mathbb{F}}_{p}$ for all $\alpha \in \mathcal{T}$ for $f \in \overline{\mathbb{F}}_{p}(\widetilde{U})$, we consider the image $X^{\circ} \subset U$ of $\{u \in \widetilde{U} \mid f(u)=0\}$ and its Zariski closure $X$. By the same argument, we have $X=V$ if $d-1>0$. Thus even in $\overline{\mathbb{F}}_{p}(\widetilde{U})$, such an $f \neq 0$ does not exist. Thus if $0 \neq f \in \widehat{\mathcal{O}}_{V, x_{0}}$ satisfies this property, $f$ is transcendental over $\mathcal{O}_{V, x_{0}}$ if $[F: \mathbb{Q}] \geq 2$. We will not use this transcendence result in the rest of the paper.

\section{EigENCOORDINATES}

Let $V$ be the geometrically irreducible component of $S h_{/ \mathcal{W} \Xi}^{(\Xi)}$ as in Theorem 2.1 containing the point $x \in V\left(\mathcal{W}_{\Xi}\right)$, and take a neat open compact subgroup $K$ of $\left.G\left(\mathbb{A}^{(} \Xi\right), \infty\right)$. As before, let $x_{0}$ be the image of $x$ in $V\left(\overline{\mathbb{F}}_{p}\right)$ (assuming $\left.p \in \Xi\right)$. Recall $V_{K}$ defined in Section 3 which is the geometrically irreducible component of $S h_{K}^{(\Xi)}$ containing the projection of $x$. Let $\mathcal{O}$ be the stalk $\mathcal{O}_{V_{K / \mathcal{W}}, x} \subset \mathcal{O}_{V_{/ \mathcal{W}_{\Xi}}, x}=\mathcal{O}_{S h^{(\Xi)}, x}$ at the $\mathcal{W}_{\Xi}$-point $x \in V\left(\mathcal{W}_{\Xi}\right)$. The $\mathcal{W}_{\Xi}$-algebra $\mathcal{O}$ is semi-local (of finite type), and its maximal ideal $\mathfrak{m}_{l, x}$ over $(l) \subset \mathcal{W}_{\Xi}$ for $l \in \Xi$ is unique. Since $V / V_{K}$ is étale, we may identify the $P$-adic localization-completion of $\mathcal{O}$ and that of $\mathcal{O}_{V, x}$ for any prime ideal $P$ of $\mathcal{O}_{V, x}$. We consider the prime $P=P_{x}$ given by the kernel of $\mathcal{O} \rightarrow \mathcal{W}_{\Xi}$ induced by evaluation at $x$. Then $P$ is a prime ideal inside the Jacobson radical of $\mathcal{O}$ (i.e., the intersection of all the maximal ideals $\left\{\mathfrak{m}_{l, x}\right\}_{l \in \Xi}$ of $\mathcal{O}$ indexed by the residual characteristic $l \in \Xi$ of $\left.\mathfrak{m}_{l, x}\right)$. Let $\mathcal{K}$ be the field of fraction of $\mathcal{W}_{\Xi}$, and consider the localization completion $\widehat{\mathcal{O}}_{P} \cong \mathcal{K}\left[\left[T_{1}, \ldots, T_{d}\right]\right]$ for $d$ variables $T_{j}$. We can extend scalars to $\mathbb{C}_{v}$ for $v=l, \infty$ by $\mathcal{K} \subset \overline{\mathbb{Q}} \stackrel{i_{v}}{\longrightarrow} \mathbb{C}_{v}$ and consider $\mathcal{O}_{v}=\mathcal{O} \otimes \mathcal{W}_{\Xi} \mathbb{C}_{v}$. Then the evaluation at $x \in V\left(\mathbb{C}_{v}\right)$ gives rise to a $\mathbb{C}_{v}$-algebra homomorphism $\mathcal{O}_{v} \rightarrow \mathbb{C}_{v}$ whose kernel we denote by $P_{v}$; thus, $P=P_{v} \cap \mathcal{O}$. Then we localize $\mathcal{O}_{v}$ at $P_{v}$ and write the localization as $\mathcal{O}_{v, P_{v}}$. We then complete it $P_{v}$-adically, getting $\widehat{\mathcal{O}}_{v, P_{v}}=\lim _{n} \mathcal{O}_{v, P_{v}} / P_{v}^{n} \mathcal{O}_{v, P_{v}}$. We then have a commutative 
diagram of inclusions

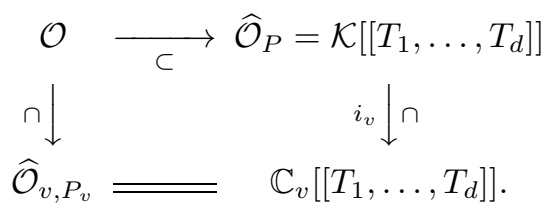

As described above, we study coordinates around a point on $\widehat{S}^{\text {ord }}$. The coordinate can be a formal function on $\widehat{S}^{\text {ord }}$ or a rigid analytic function defined on a rigid open neighborhood of the point in the associated $p$-adic rigid analytic space denoted by $\left(\widehat{S}^{\text {ord }}\right)^{p \text {-an }}$. Here is what we mean by the rigid analytic space associated to $\widehat{S}^{\text {ord }}$. A functor associating to a formal scheme $\operatorname{Spf}(A)$ a rigid analytic space is studied by Raynaud and Berthelot. The theory of Raynaud is explained in [BL], and one can find a good account of that by Berthelot in [dJ], Section 7. As a special case of his theory, Berthelot associates to $\operatorname{Spf}\left(W_{p}[[(T)]] /\left(f_{1}(T), \ldots, f_{m}(T)\right)\right)$ for a finite set of variable $(T)=\left(T_{1}, \ldots, T_{n}\right)$ and a finite set of power series $f_{j}(T) \in W[[(T)]]:=W\left[\left[T_{1}, T_{2}, \ldots, T_{n}\right]\right]$ the rigid analytic space $X^{r i g}$ given by the zero set of $\left\{f_{j}\right\}_{j}$ in the open unit disk $D^{n}$ in the $n$-dimensional affine space over the field of fraction $\operatorname{Frac}(W)$ of $W$. Here we take the base-change of $X^{\text {rig }}$ to $\mathbb{C}_{p}$ (see $\left[\mathrm{NAA}, \S 9.3 .6\right.$ ), and write it as $X_{/ \mathbb{C}_{p}}^{p \text {-an }}$. This construction appears very naive, but as exposed in $\mathrm{dJ}$, this functor has intrinsic meaning and functorial construction, and it extends naturally the results of Raynaud to formal schemes not necessarily of finite type over $W$. For $\mathfrak{p}_{\varphi} \in \Sigma^{\text {ord }}$ associated to $i_{p} \circ \varphi: F \rightarrow \mathbb{C}_{p}$, we extend $i_{p} \circ \varphi$ to a projection $W_{p} \otimes_{\mathbb{Z}} O=W_{p}^{I} \rightarrow \mathbb{C}_{p}$ (which is still denoted by $\left.i_{p} \circ \varphi\right)$, and we put $\tau_{\varphi}=\tau_{\varphi, p}=\log _{p} \circ i_{p} \circ \varphi \circ t_{\mathfrak{p}_{\varphi}}$ for the Serre-Tate coordinate $t_{\mathfrak{p}_{\varphi}}$ on $\widehat{S}_{\mathfrak{p}_{\varphi}}^{\text {ord }}=\widehat{\mathbb{G}}_{m} \otimes_{\mathbb{Z}_{p}} O_{\mathfrak{p}_{\varphi}}$. Then a convergent power series in $\mathbb{C}_{p}\left\{\left\{\tau_{\varphi}\right\}\right\}_{\varphi \in \Sigma^{\text {ord }}}$ is a rigid analytic function well defined on $\left(\widehat{S}^{\text {ord }}\right)^{p \text {-an }}$, where $\mathbb{C}_{p}\left\{\left\{\tau_{\varphi}\right\}\right\}$ is made up of power series $\sum_{n=\left(n_{\varphi}\right) \geq 0} a_{n} \tau^{n}$ in $\mathbb{C}_{p}\left[\left[\tau_{\varphi}\right]\right]_{\varphi \in \Sigma^{\text {ord }}}$ with $\lim _{|n| \rightarrow \infty}\left|a_{n}\right|_{p}=0$ for $|n|=\sum_{\varphi} n_{\varphi}$. The GAGA type theorems associating an analytic sheaf $\mathfrak{F}^{p \text {-an }}$ (again $\mathfrak{F}^{p \text {-an }}$ is the base change to $\mathbb{C}_{p}$ of $\mathfrak{F}^{\text {rig }}$ in dJ] ) to a formal coherent sheaf $\mathfrak{F}$ are given in this setting in [dJ], §7.1.11, and the analytic/formal sheaf of a Kähler differential is discussed in [d], $\left\{7.1 .12\right.$. In particular, for a rigid point $x \in X^{p \text {-an }}$ coming from a smooth formal point $x \in X(W)$ in the generic fiber, the formal cotangent space $\Omega_{X / W}(x) \otimes_{W} \mathbb{C}_{p}$ and the analytic cotangent space $\Omega_{X^{p-a n} / \mathbb{C}_{p}}(x)$ at $x$ are identical.

For an $l$-adic Barsotti-Tate $O$-module $B$ defined over a ring $C$, we write $\operatorname{End}_{O}(B / C)$ for the ring of $O$-linear endomorphisms of $B$ defined over a ring $C$ and put $\operatorname{End}_{F}^{0}(B / C)=\operatorname{End}_{O}(B / C) \otimes_{\mathbb{Z}} \mathbb{Q}$. For a finite extension $k$ of $\mathbb{Q}$ in $\overline{\mathbb{Q}}$ with integer ring $\mathfrak{O}$, put $\mathcal{W}_{k}=\mathcal{W}_{\Xi} \cap k$.

Lemma 4.1. Let $l \in \Xi$ be a prime. Let $E_{/ F}$ be a quadratic extension with $T_{E}=$ $\operatorname{Res}_{E / \mathbb{Q}} \mathbb{G}_{m}$. Suppose that $\left(A, \bar{\lambda}, \theta, \eta^{(\Xi)}\right)$ is the test object sitting over $x \in V\left(\mathcal{W}_{\Xi}\right)$ such that the image of $x$ in $V_{K}$ (for a neat open compact subgroup $K$ of $G\left(\mathbb{A}^{(\Xi), \infty)}\right.$ ) falls in $V_{K}\left(\mathcal{W}_{k}\right)$ for a finite extension $k / \mathbb{Q}$. For $\mathfrak{L}=\mathcal{W}_{k} \cap \mathfrak{m}_{l, x}$, let $\left(A_{\mathfrak{L}}, \bar{\lambda}_{\mathfrak{L}}, \theta_{\mathfrak{L}}, \eta_{\mathfrak{L}}^{(\Xi)}\right)$ be its reduction modulo $\mathfrak{m}_{l, x}$ sitting over $x_{\mathfrak{L}} \in V\left(\overline{\mathbb{F}}_{l}\right)$ (so $A_{\mathfrak{L}}$ is defined over $\mathbb{F}_{\mathfrak{L}}=$ $\mathfrak{O} / \mathfrak{L})$. Suppose that we have a level $\mathfrak{l}$-structure $\eta_{\mathfrak{l}}:\left(\mu_{l^{\infty}} \otimes_{\mathbb{Z}_{l}} O_{\mathfrak{l}}\right) \cong A\left[\mathfrak{l}^{\infty}\right]^{\circ}$ defined over $\mathcal{W}_{l}$ for a prime $\mathfrak{l} \in \Sigma_{l}^{\text {ord }}$ such that $\eta_{\mathfrak{l}} \oplus{ }^{t} \eta_{\mathfrak{l}}^{-1}:\left(\mu_{l} \infty \otimes_{\mathbb{Z}_{l}} O_{\mathfrak{l}}\right) \oplus\left(F_{\mathfrak{l}} / O_{\mathfrak{l}}\right) \cong A\left[\mathfrak{l}^{\infty}\right]$ over $W_{l}$ as Barsotti-Tate $O$-modules, where $A\left[\left[^{\infty}\right]^{\circ}\right.$ is the connected component of the Barsotti-Tate group $A\left[\mathfrak{l}^{\infty}\right] / \mathcal{W}_{l}$ and ${ }^{t} \eta_{\mathfrak{l}}^{-1}$ is the dual inverse of $\eta_{\mathfrak{l}}$ under the 
Cartier duality induced by the polarization $\lambda$. If we have embeddings $\widehat{\rho}: T_{E}(\mathbb{Q}) \rightarrow$ $G\left(\mathbb{A}^{(\Xi), \infty}\right)$ and $E \ni \alpha \mapsto \alpha_{q} \in \operatorname{End}_{F}^{0}\left(A_{\mathfrak{L}}\left[q^{\infty}\right]_{/ \mathbb{F}_{\mathfrak{L}}}\right)$ for all primes $q$ outside $\Xi$ satisfying $\eta_{\mathfrak{L}, q}^{(\Xi)} \circ \widehat{\rho}(\alpha)=\alpha_{q} \circ \eta_{\mathfrak{L}, q}^{(\Xi)}$ for all $q \notin \Xi$ for the $q$-component $\eta_{q}^{(\Xi)}$ of the level structure $\eta^{(\Xi)}$, then

(1) $E$ is a $C M$ field isomorphic to $\operatorname{End}_{F}^{0}\left(A_{\mathfrak{L} / \overline{\mathbb{F}}_{l}}\right)$,

(2) $\widehat{\rho}\left(\mathfrak{r}_{(\Xi)}^{\times}\right)\left(\mathfrak{r}_{(\Xi)}=\mathfrak{r} \otimes_{\mathbb{Z}} \mathbb{Z}_{(\Xi)}\right)$ fixes $x_{\mathfrak{L}} \in V\left(\overline{\mathbb{F}}_{l}\right)$ for an order $\mathfrak{r}$ of E maximal at $\mathfrak{l}$,

(3) we have an F-linear embedding $\widetilde{\theta}_{\mathfrak{L}}: E \hookrightarrow \operatorname{End}_{F}^{0}\left(A_{\mathfrak{L} / \mathbb{F}_{l}}\right)$ such that $\widetilde{\theta}_{\mathfrak{L}}(\alpha)$ induces $\alpha_{q}$ in the endomorphism algebra $\operatorname{End}_{F}^{0}\left(A_{\mathfrak{L}}\left[q^{\infty}\right]\right)$ for all $q \notin \Xi$,

(4) there exist an extension of $\sigma: F \hookrightarrow \overline{\mathbb{Q}}$ to $E$ (still denoted by $\sigma$ ) and a $l$-adic rigid analytic $\sigma$-coordinate $\tau_{\sigma}=\tau_{\sigma, l}$ of the rigid analytic space associated to $\widehat{S}_{l}^{\text {ord }}$ centered at $x$ in $\widehat{S}\left(W_{l}\right)$ such that $\tau_{\sigma}(x)=0$ and $\tau_{\sigma} \circ \widehat{\rho}(\alpha)=$ $i_{l}\left(\alpha^{\sigma(1-c)}\right) \tau_{\sigma}$ for each $\sigma$ inducing the place $\mathfrak{l}$ on $F$ after composing $i_{l}$.

Moreover the coordinate $\tau_{\sigma}$ as in (4) above is unique up to scalar multiples.

We call a $\sigma$-coordinate satisfying (4) above a $\widehat{\rho}$-eigen $\sigma$-coordinate.

Proof. The test object $\left(A_{\mathfrak{L}}, \bar{\lambda}_{\mathfrak{L}}, \theta_{\mathfrak{L}}\right)$ is defined over the finite field $\mathbb{F}_{\mathfrak{L}}=\mathfrak{O} / \mathfrak{L}$. Let $R^{\prime}=\operatorname{End}_{O}\left(A_{\mathfrak{L} / \mathbb{F}_{\mathfrak{L}}}\right)$. Since $\Sigma_{l}^{\text {ord }} \neq \emptyset$, by a theorem of Tate (see ABV], Appendix I, Theorem 3), $R^{\prime}$ is an order of a semi-simple quadratic extension $M^{\prime}$ of $F$ (generated by the Frobenius endomorphism over $\mathbb{F}_{\mathfrak{L}}$ ) whose simple components are all $\mathrm{CM}$ fields; thus, $M^{\prime}$ must be a CM quadratic extension (as it cannot be isomorphic to $F \times F)$. By another theorem of Tate ([ABV], Appendix I, Theorem 2), we have

$$
\operatorname{End}_{O}\left(A_{\mathfrak{L}}\left[q^{\infty}\right]_{/ \mathbb{F}_{\mathfrak{L}}}\right)=\operatorname{End}_{O\left[\operatorname{Gal}\left(\overline{\mathbb{F}}_{l} / \mathbb{F}_{\mathfrak{L}}\right)\right]}\left(T_{q} A_{\mathfrak{L}}\right)=R^{\prime} \otimes_{\mathbb{Z}} \mathbb{Z}_{q}=: R_{q}^{\prime}
$$

Since $E \hookrightarrow \operatorname{End}_{F}^{0}\left(A_{\mathfrak{L}}\left[q^{\infty}\right]_{/ \mathbb{F}_{\mathfrak{L}}}\right)$ by $\alpha \mapsto \alpha_{q}$, we have $E_{q}=E \otimes_{\mathbb{Z}} \mathbb{Q}_{q} \cong M_{q}^{\prime}$ for all primes $q$ outside $\Xi$, and we conclude to have $\widetilde{\theta}_{\mathfrak{L}}: E \cong M^{\prime}$; so, $E$ is a CM field. Put $\mathfrak{r}=\widetilde{\theta}_{\mathfrak{L}}^{-1}\left(R^{\prime}\right) \subset E$, which is an order of $E$ maximal at $\mathfrak{l}$ by Lemma 2.2 applied to $\mathfrak{l}$. Note that $E_{\mathfrak{l}} \cong F_{\mathfrak{l}} \times F_{\mathfrak{l}}$. Since $\widetilde{\theta}_{\mathfrak{L}}(\alpha)$ for $\alpha \in \mathfrak{r}_{(\Xi)}^{\times}$is a prime-to- $\Xi$ isogeny sending $\left(A_{\mathfrak{L}}, \bar{\lambda}_{\mathfrak{L}}, \eta_{\mathfrak{L}}^{(\Xi)}\right)_{/ \overline{\mathbb{F}}_{l}}$ to $\left(A_{\mathfrak{L}}, \bar{\lambda}_{\mathfrak{L}}, \widetilde{\theta}_{\mathfrak{L}}(\alpha) \circ \eta_{\mathfrak{L}}^{(\Xi)}\right)_{/ \overline{\mathbb{F}}_{l}}=\left(A_{\mathfrak{L}}, \bar{\lambda}_{\mathfrak{L}}, \eta_{\mathfrak{L}}^{(\Xi)} \circ \widehat{\rho}(\alpha)\right)_{/ \overline{\mathbb{F}}_{l}}, \widehat{\rho}(\alpha)$ fixes $x_{\mathfrak{L}}$ by the universality of $S h_{/ \overline{\mathbb{F}}_{l}}^{(\Xi)}$.

By Serre-Tate theory, we have the Serre-Tate coordinate $t:=t_{\mathfrak{l}}$ of the $\mathfrak{l}$ component $\widehat{\mathbb{G}}_{m} \otimes_{\mathbb{Z}_{l}} O_{\mathfrak{l}}$ of $\widehat{S}_{l}^{\text {ord }}$ such that an $O$-linear automorphism $a \in$ $\operatorname{Aut}_{O}\left(\widehat{\mathbb{G}}_{m} \otimes O_{\mathfrak{l} / W_{l}}\right)=O_{\mathfrak{l}}^{\times}$acts by $t \mapsto t^{a}$. As seen in [H10], Lemma 3.3, $\alpha \in$ $\operatorname{End}_{F}^{0}\left(A_{\mathfrak{L}}\right)=E$ acts on $\widehat{S}_{l}^{\text {ord }}$ by universality, and this action induces a homomorphism : $\mathfrak{r}_{(\Xi)}^{\times} \rightarrow \operatorname{Aut}_{O}\left(\widehat{\mathbb{G}}_{m} \otimes O_{\mathfrak{l} / W_{l}}\right)=O_{\mathfrak{l}}^{\times}$sending $\alpha \in E^{\times}$to $\alpha^{\sigma(1-c)} \in F_{\mathfrak{l}}$ under an extension $\sigma: E \hookrightarrow \overline{\mathbb{Q}}$ of $\sigma: F \hookrightarrow \overline{\mathbb{Q}}$ inducing $\mathfrak{l}$ and the projection of $E_{\mathfrak{l}}$ to one of its simple components isomorphic to $F_{\mathfrak{l}}$. Though the proof of [H10], Lemma 3.3 is given for ordinary $A_{\mathfrak{L}}$, the same argument applied to $A_{\mathfrak{L}}\left[l^{\infty}\right]$ (in place of $A_{\mathfrak{L}}\left[l^{\infty}\right]$ there) works equally well.

Let $\log _{l}: 1+l W_{l} \rightarrow W_{l}$ be the $l$-adic logarithm map. Write $\sigma_{\mathfrak{l}}$ for the projection of $W_{l} \otimes_{\mathbb{Z}} O \subset W_{l}^{I}$ to $W_{l}$ induced by $\sigma: O \hookrightarrow \overline{\mathbb{Q}}$. Then we get a standard $\sigma$ coordinate $\tau_{\sigma}$ as the following composition:

$$
\widehat{\mathbb{G}}_{m} \otimes_{\mathbb{Z}_{l}} O_{\mathfrak{l}}\left(W_{l}\right)=\left(1+l W_{l}\right) \otimes_{\mathbb{Z}_{l}} O_{\mathfrak{l}} \stackrel{\log _{l} \otimes 1}{\longrightarrow} W_{l} \otimes_{\mathbb{Z}_{l}} O_{\mathfrak{l}} \stackrel{\sigma_{\mathfrak{l}}}{\longrightarrow} W_{l} .
$$


We can perform the same procedure for other primes $\mathfrak{l}_{\varphi} \in \Sigma_{l}^{\text {ord }}$ and get a coordinate $\tau_{\varphi}$ for $\varphi \in I$ associated to $\mathfrak{l}_{\varphi}$ satisfying the same property above by replacing $\sigma$ with $\varphi$. The associated rigid analytic space $\left(\widehat{S}_{l}^{\text {ord }}\right)^{l \text {-an }}$ has by definition the coordinates $\left\{\tau_{\varphi}\right\}_{\varphi \in \Sigma_{l}^{\text {ord }}}$ (here $\Sigma_{l}^{\text {ord }}$ is regarded as a set of field embeddings of $F$ into $\mathbb{C}_{l}$ ). Note here that $t_{\sigma}(x)=1$ and $\tau_{\sigma}(x)=0$ (by the splitting $A\left[\mathfrak{l}^{\infty}\right] \cong$ $\left(\mu_{l \infty} \otimes_{\mathbb{Z}_{l}} O_{\mathfrak{l}}\right) \oplus\left(F_{\mathfrak{l}} / O_{\mathfrak{l}}\right)$ over $\left.W_{l}\right)$, but for $\varphi$ not associated to $\mathfrak{l}$, $\tau_{\varphi}(x)$ may not be equal to 0 . Then any other rigid analytic coordinate $f$ centered at $x$ (i.e., $f(x)=0$ ) is anyway a power series convergent on an open subset of the rigid analytic space $\left(\widehat{S}_{l}^{\text {ord }}\right)^{l \text {-an }}$ associated to $\widehat{S}_{l}^{\text {ord }}$. Therefore it has power series expansion $f(\tau)=\sum_{\nu} a_{\nu} \tau^{\nu}$ for $\tau^{\nu}=\prod_{\varphi \in \Sigma_{l}^{\text {ord }}} \tau_{\varphi}^{\nu_{\varphi}}$. If $f \circ \widehat{\rho}(\alpha)=i_{l}\left(\alpha^{\sigma(1-c)}\right) f$, by equating the power series expansion, we find that $f$ is a constant multiple of $\tau_{\sigma}$.

If $v=\infty$, we put $G_{\infty}=\operatorname{Aut}_{F}\left(\mathfrak{H}^{I}\right)$ (the complex analytic automorphism group inducing an $O$-linear map on $\left.\Omega_{\mathfrak{H}^{I} / \mathbb{C}}\right)$; thus, $G_{\infty}=P S L_{2}\left(F_{\mathbb{R}}\right)=\prod_{\varphi \in I} P S L_{2}(\mathbb{R})$ according to the decomposition $F_{\mathbb{R}}=F \otimes_{\mathbb{Q}} \mathbb{R}=\prod_{\varphi \in I} \mathbb{R}$ for which the projection to the $\varphi$-factor induces $i_{\infty} \circ \varphi: F \rightarrow \mathbb{R}$ (e.g., [DLS], Chapter IV). As is well known, the stabilizer of $x \in V\left(\mathcal{W}_{\Xi}\right)$ in $G_{\infty}$ is given by an anisotropic torus in $G_{\infty}$. Write the torus as $\mathcal{T}_{\infty, x}=\mathcal{T}_{x}=\prod_{\varphi \in I} \mathcal{T}_{\varphi, x}$ for $\mathcal{T}_{\varphi, x} \cong \mathbb{C}^{\times} / \mathbb{R}^{\times}$. We put $\Sigma_{\infty}^{\text {ord }}:=I$.

If $v=p$ and $\Sigma_{p}^{\text {ord }} \neq \emptyset$, we write $G_{p}=\operatorname{Aut}_{F}\left(\widehat{S}^{\text {ord }}\right)$ (the group of formal automorphisms over $W_{p}$ compatible with $O_{(\Xi)}^{\times}$-action on $\left.\Omega_{\widehat{S}_{\text {ord }} / W_{p}}\right)$. Note that $\widehat{S}^{\text {ord }} \cong \widehat{\mathbb{G}}_{m} \otimes_{\mathbb{Z}_{p}} O_{\Sigma^{\text {ord }}}$ as seen in Section 1. We fix once and for all the identification $\widehat{S}^{\text {ord }}=\widehat{\mathbb{G}}_{m} \otimes_{\mathbb{Z}_{p}} O_{\Sigma^{\text {ord }}}$. This is tantamount to fixing an isomorphism $\mu_{p} \infty \otimes_{\mathbb{Z}_{p}} O_{\mathfrak{p}} \cong$ $A_{0}\left[\mathfrak{p}^{\infty}\right]^{\circ}$ for each $\mathfrak{p} \in \Sigma_{p}^{\text {ord }}$, so, as before, we write $t_{\mathfrak{p}}$ for the Serre-Tate (multiplicative) coordinate of $\widehat{S}_{\mathfrak{p}}^{\text {ord }}$. We have $\operatorname{Aut}_{S C H}\left(\mathbb{G}_{m}\right)=\operatorname{Aut}_{S C H}\left(\operatorname{Spec}\left(W_{p}\left[t, t^{-1}\right]\right)\right)=$ $\mathbb{G}_{m}\left(W\left[t, t^{-1}\right]\right)=W\left[t, t^{-1}\right]^{\times}$(the scheme automorphism group). Similarly, writing $t=1+T$, we have Aut $\left(\widehat{\mathbb{G}}_{m}\right)=\operatorname{Aut}\left(\operatorname{Spf}\left(W_{p}[[T]]\right)\right)=\widehat{\mathbb{G}}_{m}(W[[T]])=1+\mathfrak{m}_{W[[T]]}$ for the maximal ideal $\mathfrak{m}_{W[[T]]}$ of $W[[T]]$. Here Aut $\left(\widehat{\mathbb{G}}_{m}\right)$ stands for the automorphism group of the formal scheme $\widehat{\mathbb{G}}_{m}$. Note that $\widehat{\mathbb{G}}_{m} \otimes_{\mathbb{Z}_{p}} O_{\Sigma_{\text {ord }}}=\operatorname{Spf}\left(W\left[\left[O_{\Sigma^{\text {ord }}}\right]\right]\right)=$ $\operatorname{Spf}\left(W\left[\left[T_{\varphi}\right]\right]_{\varphi \in \Sigma_{\text {ord }}}\right)$, where $T_{\varphi}=t_{\varphi}-1$ for the Serre-Tate $\varphi$-coordinate $t_{\varphi}$. If $\phi \in G_{p}$, then $\phi(T)=\left(\phi_{\varphi}(T)\right)_{\varphi \in \Sigma^{\text {ord }}}$; so, we have $\phi^{*} d T_{\varphi}=\sum_{\psi} \frac{\partial \phi_{\varphi}}{\partial T_{\psi}} d T_{\psi}$. Then having $\phi^{*}\left(d T_{\varphi}\right) \in \mathcal{O}_{\widehat{S}} d T_{\varphi}$ implies $\frac{\partial \phi_{\varphi}}{\partial T_{\psi}}=0$ if $\varphi \neq \psi$. Thus $\phi_{\varphi}$ only involves the parameter $T_{\varphi}$, so, $G_{p}=\widehat{\mathbb{G}}_{m}(W[[T]])^{\Sigma^{\text {ord }}}$ by sending $\phi$ to $\left(\phi_{\varphi}\left(T_{\varphi}\right)\right)_{\varphi \in \Sigma^{\text {ord }}}$. Let $\mathbf{1} \in \widehat{S}^{\text {ord }}$ be the $W_{p}$-point given by $t_{\varphi}=1$ for all $\varphi \in \Sigma^{\text {ord }}$ (i.e., the origin). The point $x \in V\left(\mathcal{W}_{\Xi}\right)$ gives rise to $x \in \widehat{S}^{\text {ord }}\left(W_{p}\right)$ and $x \in\left(\widehat{S}^{\text {ord }}\right)^{p \text {-an }}\left(\mathbb{C}_{p}\right)$. The group of $O$-linear automorphisms $\mathcal{T}_{\mathbf{1}}=\mathcal{T}_{p, \mathbf{1}}:=\operatorname{Aut}_{O-\operatorname{lin}}\left(\widehat{S}^{\text {ord }}\right)$ is in the stabilizer of $\mathbf{1}$. The translation automorphism $T_{x}: y \mapsto x y \in \widehat{S}^{\text {ord }}$ is an element in $G_{p}$. Thus we can translate the group structure via $T_{x}$ so that $x$ becomes the identity element. Then we have $\mathcal{T}_{p, x}=\mathcal{T}_{x}:=T_{x} \mathcal{T}_{1} T_{x}^{-1} \subset G_{p}$ fix $x$. We again have $\mathcal{T}_{p, x}=\prod_{\mathfrak{p} \in \Sigma_{p}^{\text {ord }}} \mathcal{T}_{\mathfrak{p}, x}$ with $\mathcal{T}_{\mathfrak{p}, x} \cong O_{\mathfrak{p}}^{\times}$which preserve the $\mathfrak{p}$-component $\widehat{S}_{\mathfrak{p}}^{\text {ord }}:=\widehat{\mathbb{G}}_{m} \otimes_{\mathbb{Z}_{p}} O_{\mathfrak{p}} \subset \widehat{S}^{\text {ord }}$.

Lemma 4.2. Suppose that $p \in \Xi$ is unramified in $F$. If $g \in G\left(\mathbb{A}^{(\Xi), \infty)}\right.$ leaves stable $\widehat{S}_{\mathfrak{p}}^{\text {ord }}$ inducing an automorphism of formal schemes and fixes the $\mathfrak{p}$-component $x_{\mathfrak{p}} \in \widehat{S}_{\mathfrak{p}}^{\text {ord }}$ of $x$, then the action of $g$ on $\widehat{S}_{\mathfrak{p}}^{\text {ord }}$ belongs to $\mathcal{T}_{\mathfrak{p}, x}$. 
Proof. The action of $g$ on each test object $\left(A_{s}, \eta_{s}^{(\Xi)}\right)$ at $s$ is given by

$$
\left(A_{s}, \eta_{s}^{(\Xi)}\right) \mapsto\left(A_{s g}, \eta_{s g}^{(\Xi)}\right) \underset{\sim}{\stackrel{\alpha}{\sim}}\left(A_{s}, \eta_{s}^{(\Xi)} \circ g\right)
$$

with an $O$-linear prime to $\Xi$-isogeny $\alpha$. Thus it brings the $O$-linear group structure on the deformation space $\widehat{S}_{x}^{\text {ord }}\left(\right.$ of $\left(A_{x}, \eta_{x}^{(\Xi)}\right)$ ) with identity $x$ to the $O$-linear group structure on $\widehat{S}_{x g}^{\text {ord }}$ with identity $x g$ just by the construction of the Serre-Tate coordinate; in other words, we have the commutative diagram of the extensions

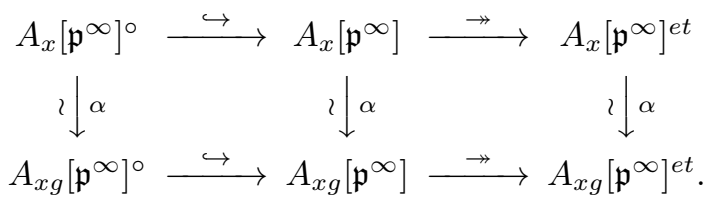

The vertical arrows are $O$-linear isomorphisms as $\alpha$ is prime to $\Xi$-isogeny. Thus if $g$ leaves $\widehat{S}_{\mathfrak{p}}^{\text {ord }}$ stable and fixes $x_{\mathfrak{p}}$, it must preserve the $O$-linear group structure of $\widehat{S}_{\mathfrak{p}}^{\text {ord }}$ with identity $x_{\mathfrak{p}}$. This shows that the action of $g$ on $\widehat{S}_{\mathfrak{p}}^{\text {ord }}$ belongs to $\mathcal{T}_{\mathfrak{p}, x}$.

Since the stabilizer of the $\varphi$-component $x_{\varphi}$ of $x$ in $\mathfrak{H}^{I}$ is $\mathcal{T}_{\varphi, x}$, we have immediately

Lemma 4.3. If $g \in G\left(\mathbb{A}^{(\Xi)}\right)$ leaves stable the $\varphi$-component $\mathfrak{H}_{\varphi}=\mathfrak{H}$ of $\mathfrak{H}^{I}$ and fixes the $\varphi$-component $x_{\varphi} \in \mathfrak{H}_{\varphi}$, then the action of $g$ on $\mathfrak{H}_{\varphi}$ belongs to $\mathcal{T}_{\varphi, x}$.

Lemma 4.4. Let $x \in V\left(\mathcal{W}_{\Xi}\right)$, and suppose that $v \in \Xi \cup\{\infty\}$ is unramified in $F$. Then for each $\varphi \in \Sigma_{v}^{\text {ord }}$, there always exists a $\varphi$-coordinate $\tau_{\varphi}=\tau_{\varphi, v}$ with $\tau_{\varphi}(x)=0$ convergent on an open rigid affinoid neighborhood $D_{x}\left(\tau_{\varphi}\right)$ of $x$ satisfying $\tau_{\varphi} \circ a=a_{\mathfrak{v}}^{\varphi} \tau_{\varphi}$ for $a=\left(a_{\mathfrak{v}}\right)_{\mathfrak{v} \in \Sigma_{v}^{\text {ord }}} \in \mathcal{T}_{v, x}=O_{\Sigma_{v}^{\text {ord }}}^{\times}$if $v<\infty$ and $\tau_{\varphi} \circ a=a_{\varphi} \tau_{\varphi}$ for $a \in \mathcal{T}_{\infty, x}=\left(\mathbb{C}^{\times} / \mathbb{R}^{\times}\right)^{I}$ if $v=\infty$, where $\mathfrak{v}$ is the place of $F$ induced by $i_{v} \circ \varphi: F \hookrightarrow$ $\mathbb{C}_{p}$. The coordinate $\tau_{\varphi, v}$ is unique up to scalar multiple.

If $x_{\mathfrak{p}} \neq \mathbf{1}_{\mathfrak{p}} \in \widehat{S}_{\mathfrak{p}}^{\text {ord }}$, the automorphism $\phi \in \mathcal{T}_{p, x} \cap \operatorname{Aut}\left(\widehat{S}_{\mathfrak{p}}^{\text {ord }}\right)$ may not extend to the global $V$ as the translation $T_{x_{\mathfrak{p}}} \neq 1$ is possibly a transcendental action only defined on the formal scheme $\widehat{S}_{\mathfrak{p}}^{\text {ord }}=\widehat{\mathbb{G}}_{m} \otimes_{\mathbb{Z}_{p}} O_{\mathfrak{p}}$. Therefore, $\tau_{\sigma, p}$ in the lemma may not be a $\widehat{\rho}$-eigen coordinate for any choice of $\widehat{\rho}$.

Proof. The result for $v=\infty$ is well known (see the argument towards the end of the proof of Theorem 4.5). If $v<\infty$, by Serre-Tate deformation theory, we have $t_{\mathfrak{v}}$ centered at 1 such that $t_{\mathfrak{v}} \circ a=t_{\mathfrak{v}}^{a}$ for $a \in \mathcal{T}_{\mathbf{1}}$. Then $\tau_{\varphi, v}:=\log _{p} \circ \varphi_{\mathfrak{v}} \circ t_{\mathfrak{v}} \circ T_{x}^{-1}$ does the job, where $\varphi_{\mathfrak{v}}: W_{v} \otimes_{\mathbb{Z}} O=W_{v}^{I} \rightarrow \mathbb{C}_{v}$ is the $\varphi$-projection. Uniqueness up to scalar multiple is clear from $\tau_{\varphi} \circ a=a_{\mathfrak{v}}^{\varphi_{\mathfrak{v}}} \tau_{\varphi}$.

Since $\left(A, \lambda, \theta, \bar{\eta}^{(\Xi)}\right)$ gives the point $x \in V\left(\mathcal{W}_{\Xi}\right)$, we may think of its $p$-fiber

$$
\left(A_{0}, \bar{\lambda}_{0}, \theta_{0}, \eta_{0}^{(\Xi)}\right)_{/ \overline{\mathbb{F}}_{p}}=\left(A, \lambda, \theta, \bar{\eta}^{(\Xi)}\right) \times_{\mathcal{W}_{\Xi}} \overline{\mathbb{F}}_{p} .
$$

Let $M=\operatorname{End}^{0}\left(A_{0 / \overline{\mathbb{F}}_{p}}\right)$, which is a CM quadratic extension of $F$ in which $\mathfrak{p}$ splits by Lemma 2.2. As before, we define $\widehat{\rho}: T_{M}\left(\mathbb{Z}_{(\Xi)}\right) \rightarrow G\left(\mathbb{A}^{(\Xi), \infty)}\right.$ by $\tilde{\theta}_{\mathfrak{P}}(\alpha) \circ \eta_{0}^{(\Xi)}=$ $\eta_{0}^{(\Xi)} \circ \widehat{\rho}(\alpha)$.

Theorem 4.5. Let the notation be as above. Let $i_{p} \circ \sigma$ (resp. $\left.i_{l} \circ \sigma\right)$ induce $\mathfrak{p} \in \Sigma_{p}^{\text {ord }}$ (resp. $\mathfrak{l} \in \Sigma_{l}^{\text {ord }}$ ) for $p, l \in \Xi$ for $\left(A, \bar{\lambda}, \theta, \eta^{(\Xi)}\right)$ sitting over $x \in V\left(\mathcal{W}_{\Xi}\right)$. Assume that $p$ and $l$ are unramified in $F_{/ \mathbb{Q}}$, and suppose that we have a p-analytic $\widehat{\rho}$-eigen $\sigma$-coordinate $\tau_{p}=\tau_{\sigma, p}$ of the form $\tau_{p}=\log _{p} \circ \sigma_{\mathfrak{p}} \circ t_{\mathfrak{p}}$ with $\tau_{p}(x)=0$ around 
$x \in V\left(\mathcal{W}_{\Xi}\right) \subset V\left(\mathbb{C}_{p}\right)$, where $\sigma_{\mathfrak{p}}: W_{p} \otimes_{\mathbb{Z}} O=W_{p}^{I} \rightarrow \mathbb{C}_{p}$ is the $\sigma$-projection. Then for $v \in\{p, l, \infty\}=: \Xi_{0}$, we have a $v$-analytic $\widehat{\rho}$-eigen $\sigma$-coordinate coordinate $\tau_{v}=\tau_{\sigma, v}$ with $\tau_{v}(x)=0$. Identifying $\mathbb{C}_{p}=\mathbb{C}_{l}=\mathbb{C}$ (as in the introduction), in the formal completion of $\mathcal{O}_{V_{/ \mathbb{C}_{v}}, x}$ along the $\mathbb{C}_{v}$-point $x \in V\left(\mathbb{C}_{v}\right)$, the coordinates $\left\{\tau_{v}\right\}_{v \in \Xi_{0}}$ are proportional (i.e., they are a non-zero constant multiple of each other).

Write $\left(\underline{\omega}^{2 \sigma}\right)^{v \text {-an }}$ for the $v$-analytic line bundle on $\left(\widehat{S}^{\text {ord }}\right)^{v \text {-an }}$ coming from $\underline{\omega}^{2 \sigma}$ by a $v$-analytic version of GAGA theorems (e.g., dJ], $§ 7.1 .11$ and $\mathrm{C}$, Example 2.4.6). We write $\mathfrak{v}$ for $\mathfrak{l}$ and $\mathfrak{p}$ according to $v=l$ and $p$, and $\Sigma_{\mathfrak{v}}^{\text {ord }}$ for the set of all embeddings $\sigma: F \rightarrow \overline{\mathbb{Q}}$ such that $i_{v} \circ \sigma$ induces $\mathfrak{v}$. To avoid confusion, if necessary, we write the $v$-adic deformation space $\widehat{S}$ of $A \otimes \mathcal{W}_{v} \overline{\mathbb{F}}_{v}$ as $\widehat{S}_{v}$.

Proof. Since $\widehat{S}_{p}=\widehat{S}_{p}^{\text {ord }} \times \widehat{S}_{p}^{l l}$ (see Proposition 1.2$)$, the co-tangent space $\Omega_{(\widehat{S})^{p \text {-an }} / \mathbb{C}_{p}}(x)$ at $x$ of $(\widehat{S})^{p \text {-an }}$ is a direct sum of $\Omega_{\left(\widehat{S}^{\text {ord }}\right)^{p \text {-an }} / \mathbb{C}_{p}}\left(x^{\text {ord }}\right)$ and $\Omega_{\left(\widehat{S}^{l l}\right)^{p \text {-an }} / \mathbb{C}_{p}}\left(x^{l l}\right)$ for the projection $x^{\text {? }}$ of $x$ to $\left(\widehat{S}^{?}\right)^{p \text {-an }}$. Then we can further decompose

$$
\Omega_{\left(\widehat{S}^{\text {ord }}\right)^{p-\operatorname{an}} / \mathbb{C}_{p}}\left(x^{\text {ord }}\right)=\bigoplus_{\mathfrak{p}_{\varphi} \in \Sigma^{\text {ord }}} \Omega_{\left(\widehat{S}_{\mathfrak{p}_{\varphi}}^{\text {ord }}\right)^{p-\operatorname{an}} / \mathbb{C}_{p}}\left(x_{\mathfrak{p}_{\varphi}}\right)
$$

and

$$
\Omega_{\left(\widehat{S}_{\mathfrak{p}_{\varphi}}^{\text {ord }}\right)^{p \text {-an }} / \mathbb{C}_{p}}\left(x_{\mathfrak{p}_{\varphi}}\right)=\bigoplus_{\varphi \sim \mathfrak{p}_{\varphi}}\left(\underline{\omega}^{2 \varphi}\right)^{p \text {-an }}(x),
$$

where $x_{\mathfrak{p}_{\varphi}}$ is the projection of $x$ to $\widehat{S}_{\mathfrak{p}_{\varphi}}^{\text {ord }}$ and where in the second identity $\varphi$ runs over $\varphi \in I$ such that $i_{p} \circ \varphi$ induces the $p$-adic place $\mathfrak{p}_{\varphi}$.

Since $\tau_{p}(x)=0$, the Serre-Tate $\mathfrak{p}$-coordinate $t_{\mathfrak{p}}$ is equal to 1 at $x$. Thus,

$$
A\left[\mathfrak{p}^{\infty}\right] \cong\left(\mu_{p^{\infty}} \otimes_{\mathbb{Z}_{p}} O_{\mathfrak{p}}\right) \oplus\left(F_{\mathfrak{p}} / O_{\mathfrak{p}}\right)
$$

as Barsotti-Tate $O$-modules over $W_{p}$. Then the group $T_{M}\left(\mathbb{Z}_{(\Xi)}\right)$ acts via $\widehat{\rho}$ on the $\mathfrak{p}$-component $\widehat{S}_{\mathfrak{p}}^{\text {ord }}$, and $T_{M}\left(\mathbb{Z}_{(\Xi)}\right)$ fixes the $\mathfrak{p}$-component $x_{\mathfrak{p}}$ (this fact of $T_{M}\left(\mathbb{Z}_{(\Xi)}\right)$ fixing $x_{\mathfrak{p}}$ might not be true for $\left.\mathfrak{p}_{\varphi} \neq \mathfrak{p}\right)$. Here $\left(\underline{\omega}^{2 \sigma}\right)^{p \text {-an }}(x)$ is the $\sigma$-eigenspace as $O$-modules in $\Omega_{\left(\widehat{S}_{\mathfrak{p}}^{\text {ord }}\right)^{p \text {-an }} / \mathbb{C}_{p}}\left(x_{\mathfrak{p}}\right)$. Since $T_{M}\left(\mathbb{Z}_{(\Xi)}\right)$ fixes $x_{\mathfrak{p}}, \quad\left(\underline{\omega}^{2 \sigma}\right)^{p \text {-an }}(x) \subset$ $\Omega_{\left(\widehat{S}_{\mathfrak{p}}^{\text {ord }}\right)^{p \text {-an }} / \mathbb{C}_{p}}\left(x_{\mathfrak{p}}\right)$ for any $\sigma$ associated with $\mathfrak{p}$ is the $i_{p}\left(\alpha^{\sigma(1-c)}\right)$-eigenspace of $\widehat{\rho}(\alpha)$ $\left(\alpha \in T_{M}\left(\mathbb{Z}_{(\Xi)}\right)\right)$ in $\Omega_{\left(\widehat{S}_{\mathfrak{p}}^{\text {ord }}\right)^{p \text {-an }} / \mathbb{C}_{p}}\left(x_{\mathfrak{p}}\right)$ for an extension $\sigma: M \hookrightarrow \overline{\mathbb{Q}}$ of $\sigma: F \hookrightarrow \overline{\mathbb{Q}}$. Taking the canonical lift $A_{1} / \mathcal{W}_{\Xi}$ of $A_{0 / \mathbb{F}_{p}}$ sitting over $x_{1} \in V\left(\mathcal{W}_{\Xi}\right)$, the extension $\sigma$ is determined by complex multiplication by $M$ on $\underline{\omega}^{\sigma}\left(x_{1}\right) / \mathcal{W} \equiv$ which is the $\sigma$-eigenspace (under real multiplication by $F$ ) of $H^{0}\left(A_{1}, \Omega_{A_{1} / \mathcal{W} \Xi}\right)$ canonically isomorphic to $\underline{\omega}^{\sigma}(x)$ after extending scalars to $W_{p}$. Thus for $\sigma$ with $\mathfrak{p}=\mathfrak{p}_{\sigma}, T_{M}\left(\mathbb{Z}_{(\Xi)}\right)$ acts on $\left(\underline{\omega}^{2 \sigma}\right)^{p \text {-an }}(x)$ via the algebraic character $i_{p} \circ \sigma(1-c) \in X^{*}\left(T_{M}\right)$. Since $\left(\underline{\omega}^{2 \sigma}\right)^{p \text {-an }}(x)=\underline{\omega}^{2 \sigma}(x) \otimes \mathcal{W} \Xi \mathbb{C}_{p}$ for the algebraic co-tangent space $\underline{\omega}^{2 \sigma}(x) / \mathcal{W} \Xi$ and the action of $\widehat{\rho}$ on the rigid analytic line bundle $\left(\underline{\omega}^{2 \sigma}\right)^{p \text {-an }}$ is induced by its action on the algebraic line bundle $\underline{\omega}^{2 \sigma}$ over $\mathcal{W}_{\Xi}$, the action via $\widehat{\rho}$ must preserve the algebraic $\underline{\omega}^{2 \sigma}(x)$. Therefore it also preserves

$$
\left(\underline{\omega}^{2 \sigma}\right)^{v \text {-an }}(x)=\underline{\omega}^{2 \sigma}(x) \otimes_{\mathcal{W} \Xi} \mathbb{C}_{v}
$$

for every $v \in\{p, l, \infty\}$. Since the action of $\widehat{\rho}\left(T_{M}\left(\mathbb{Z}_{(\Xi)}\right)\right)$ on the $v$-analytic $\sigma$-cotangent bundle $\left(\underline{\omega}^{2 \sigma}\right)^{v \text {-an }}$ over $V\left(\mathbb{C}_{v}\right)$ preserves its fiber over $x, \widehat{\rho}\left(T_{M}\left(\mathbb{Z}_{(\Xi)}\right)\right)$ fixes $x_{\mathfrak{v}}$, reversing our argument. 
We now see this fact of $\widehat{\rho}\left(T_{M}\left(\mathbb{Z}_{(\Xi)}\right)\right)$ fixing $x_{\mathfrak{v}}$ group-theoretically. Because of the $v$-adic Lie group structure on $\widehat{S}_{\mathfrak{v}}^{\text {ord }}$ for $v=p, l$, the tangent space of $\widehat{S}_{\mathfrak{v}}^{\text {ord }}$ at the origin

$$
\operatorname{Tan}_{\mathfrak{v}}:=\bigoplus_{\varphi \sim \mathfrak{v}} \operatorname{Tan}_{\varphi, \mathfrak{v}} \text { for } \operatorname{Tan}_{\varphi, \mathfrak{v}}=\operatorname{Hom}_{W_{v}}\left(\underline{\omega}^{2 \varphi}(x) \otimes \mathcal{W}_{\Xi} W_{v}, W_{v}\right)
$$

is a Lie algebra over $W_{v}$, and we have the $v$-adic Lie algebra $L_{\mathfrak{v}}:=\operatorname{Lie}\left(\widehat{S}_{\mathfrak{v}}^{\text {ord }}\right)$ over $\mathbb{Z}_{v}$ canonically inside the tangent space $\operatorname{Tan}_{\mathfrak{v}}$; i.e., we have

$$
L_{\mathfrak{v}} \otimes_{\mathbb{Z}_{v}} W_{v}=\operatorname{Tan}_{\mathfrak{v}} .
$$

Since $\widehat{S}_{v}^{\text {ord }}=\widehat{\mathbb{G}}_{m} \otimes_{\mathbb{Z}_{v}} O_{\mathfrak{v}}$, the Lie algebra $L_{\mathfrak{v}}$ is isomorphic to the formal co-character group

$$
X_{*}\left(\widehat{S}_{\mathfrak{v}}^{\text {ord }}\right):=\operatorname{Hom}_{\text {formal group }}\left(\widehat{S}_{\mathfrak{v}}^{\text {ord }}, \widehat{\mathbb{G}}_{m}\right) \cong O_{\mathfrak{v}}
$$

and hence the action via $\widehat{\rho}$ preserves $L_{\mathfrak{v}}$ and the action via $\widehat{\rho}$ on $\operatorname{Tan}_{\mathfrak{v}}$ is the scalar extension of the action on $L_{\mathfrak{v}}$ to $\operatorname{Tan}_{\mathfrak{v}}$. We have the $v$-adic exponential map $\exp _{v}$ : $L_{\mathfrak{v}} \rightarrow \widehat{S}_{\mathfrak{v}}^{\text {ord }}$ (from the $v$-adic formal Lie algebra into the $v$-adic formal Lie group), which is equivariant under the action of $\widehat{\rho}$. Thus the $\mathfrak{v}$-component $\widehat{S}_{\mathfrak{v}}^{\text {ord }}$ is stable under the action of $T_{M}$ via $\widehat{\rho}$, and the fixed point in $\widehat{S}_{\mathfrak{v}}^{\text {ord }}$ of the action is the $\mathfrak{v}$ component $x_{\mathfrak{v}}$ of $x$. The action of $\alpha \in T_{M}\left(\mathbb{Z}_{(\Xi)}\right)$ on $\operatorname{Tan}_{\sigma, \mathfrak{v}}$ is via multiplication by $i_{v}\left(\alpha^{\sigma(1-c)}\right)$ for a suitable extension of $\sigma$ to $M$ (still denoted by $\left.\sigma\right)$. Thus its action on $L_{\mathfrak{v}}$ is via multiplication by $\alpha^{1-c}=\lim _{n}\left(\alpha^{1-c} \bmod \mathfrak{v}^{n}\right) \in O_{\mathfrak{v}}$, so, we can find a linear coordinate $T_{\sigma, \mathfrak{v}}$ of $\operatorname{Tan}_{\sigma, \mathfrak{v}}$ with $T_{\sigma, \mathfrak{v}} \circ \widehat{\rho}(\alpha)=\alpha^{\sigma(1-c)} T_{\sigma, \mathfrak{v}}$. Its image under an exponential map gives rise to the coordinate $t_{\sigma}$ on $\widehat{S}_{\mathfrak{v}}^{\text {ord }}$ (with $t_{\sigma} \circ \widehat{\rho}(\alpha)=t_{\sigma}^{\alpha^{\sigma(1-c)}}$ and $t_{\sigma}(x)=1$ ) whose $v$-adic logarithm is proportional to the $v$-adic logarithm of the Serre-Tate $\sigma$-coordinate. Then $\tau_{v}=\tau_{\sigma, \mathfrak{v}}:=\log _{v} \circ t_{\sigma}$ is the desired $\widehat{\rho}$-eigen $\sigma$ coordinate and $x_{\mathfrak{v}}$ is the fixed point of $\widehat{\rho}$ on $\widehat{S}_{\mathfrak{v}}^{\text {ord }}$, which induces a homomorphism $\rho_{\mathfrak{v}}: T_{M}\left(\mathbb{Z}_{(\Xi)}\right) \rightarrow \operatorname{Aut}_{O \text {-lin }}\left(\widehat{S}_{\mathfrak{v}}^{\text {ord }}\right)=\mathcal{T}_{\mathfrak{v}, x}$. To see $\tau_{v}(x)=0$ in more down-to-earth terms, note that $\mathcal{T}_{\mathfrak{v}, x}=O_{\mathfrak{v}}^{\times}$and $\rho_{\mathfrak{v}}(\alpha)=\alpha^{\sigma(1-c)} \in O_{\mathfrak{v}}^{\times}$. Since the image of $\rho_{\mathfrak{v}}$ is $\mathfrak{v}$-adically dense, by the continuity of the action, we have $\tau_{\sigma, \mathfrak{v}} \circ a=a \tau_{\sigma, \mathfrak{v}}$ for all $a \in O_{\mathfrak{v}}^{\times}=\mathcal{T}_{\mathfrak{v}, x}$. Then by Lemma 4.4, $\tau_{\sigma, \mathfrak{p}}$ and $\tau_{\sigma, \mathfrak{l}}$ are proportional to each other as formal functions on the formal completion of $V_{K / \mathbb{C}_{v}}$ along $x \in V_{K}\left(\mathbb{C}_{v}\right)$ identifying $\mathbb{C}_{v}=\mathbb{C}_{l}=\mathbb{C}_{p}$. In particular $\tau_{\sigma, \mathfrak{v}}(x)=0$, and this reconfirms that the coordinate $\tau_{v}:=\tau_{\sigma, \mathfrak{v}}$ is the desired one (up to scalar multiple).

The proof for $v=\infty$ is almost identical to the above proof for finite places. Here it is. We can bring $\mathfrak{H}^{I}$ isomorphically onto $\mathfrak{D}^{I}$ for the unit open disk $\mathfrak{D}$ in $\mathbb{C}$ by a linear fractional transformation by a matrix in $S L_{2}(\mathbb{C})^{I}$ so that $x$ is sent to the origin 0 , and the action of the torus $\mathcal{T}_{\infty, x / \mathbb{R}}$ in $P S L_{2}(\mathbb{R})_{/ \mathbb{R}}^{I}$ fixing $x$ (isomorphic to $\left.\left(\mathbb{C}^{\times} / \mathbb{R}^{\times}\right)^{I}\right)$ on the coordinate $w_{\sigma}$ of $\mathfrak{D}$ at $\sigma \in I$ is given by $w_{\varphi} \circ \alpha=\alpha_{\varphi}^{1-c} w_{\varphi}$ for $\alpha=\left(\alpha_{\varphi}\right)_{\varphi \in I} \in\left(\mathbb{C}^{\times}\right)^{I}$ (under the identification $\left.\mathcal{T}_{x}(\mathbb{C})=\left(\mathbb{C}^{\times} / \mathbb{R}^{\times}\right)^{I}\right)$. Here $\mathfrak{D}$ is the symmetric domain of $S U(1,1)$ (the signature $(1,1)$ special unitary group) isomorphic to $S L_{2}(\mathbb{R})$, and $T_{1}(\mathbb{C}) \subset S L_{2}(\mathbb{R})^{I}$ is identified with the diagonal torus in $S U(1,1)^{I}$. To explain this in down-to-earth terms, write $z$ for the standard coordinate of $\mathfrak{H}^{I} \subset \mathbb{C}^{I}$. If $\xi=z_{\varphi}(x)$, the holomorphic isomorphism $\mathfrak{H} \ni z=$ $\left(z_{\varphi}\right)_{\varphi} \mapsto w=\left(w_{\varphi}\right)_{\varphi}=\left(\frac{z_{\varphi}-\xi}{z_{\varphi}-\bar{\xi}}\right)_{\varphi} \in \mathfrak{D}$ does the job. Though $\mathfrak{D}$ is not a Lie group, it is an open neighborhood of the identity of the Lie group $\mathbb{C}^{\times}=\mathbb{G}_{m}(\mathbb{C})$, and it is a Lie semi-group. Then the argument using the tangent space at $x$ as above 
(identifying it with the Lie algebra of $\mathcal{T}_{\infty, x}$ ) done for finite places $v=p, l$ is valid also for $v=\infty$ without any change. Thus we can take $w_{\sigma}$ to be $\tau_{\sigma, \infty}=\tau_{\infty}$. Again it is proportional to $\tau_{v}$ over the formal completion.

Corollary 4.6. Let the notation and the assumption be as in Theorem 4.5. Let $\mathfrak{l} \in \Sigma_{l}^{\text {ord }}$ for a prime $l \in \Xi$ different from $p$. If the prime $\mathfrak{l}$ in $F$ is induced by $i_{l} \circ \sigma$, we have $\operatorname{End}_{F}^{0}\left(A \otimes_{\mathcal{W} \Xi} \overline{\mathbb{F}}_{l}\right) \cong M \cong \operatorname{End}_{F}^{0}\left(A \otimes_{\mathcal{W}_{\Xi}} \overline{\mathbb{F}}_{p}\right)$ as $F$-algebras (via $\left.\theta\right)$.

Proof. Let $A_{\mathfrak{L}}=A \otimes \mathcal{W}_{\Xi} \overline{\mathbb{F}}_{l}$, and write $M^{\prime}=\operatorname{End}_{F}^{0}\left(A_{\mathfrak{L}}\right)$ with $R^{\prime}=\operatorname{End}_{O}\left(A_{\mathfrak{L}}\right)$ and $M=\operatorname{End}_{F}^{0}\left(A_{0}\right)$ with $R=\operatorname{End}_{O}\left(A_{0}\right)$. Fix a level l-structure $\eta_{\mathfrak{l}}: \mu_{l \infty} \otimes_{\mathbb{Z}_{l}} O_{\mathfrak{l}} \cong$ $A\left[\left[^{\infty}\right]^{\circ}\right.$. By Theorem 4.5, we have $\tau_{l}(x)=0$; thus, $t_{\mathfrak{l}}(x)=1$, which implies that we get an $O$-linear isomorphism of Barsotti-Tate $O$-modules over $W_{l}$ :

$$
\eta_{\mathfrak{l}} \oplus^{t} \eta_{\mathfrak{l}}^{-1}:\left(\mu_{l \infty} \otimes_{\mathbb{Z}_{l}} O_{\mathfrak{l}}\right) \oplus\left(F_{\mathfrak{l}} / O_{\mathfrak{l}}\right) \cong A\left[\mathfrak{r}^{\infty}\right]
$$

Again by Theorem 4.5 we have $\tau_{l} \circ \widehat{\rho}(\alpha)=i_{l}\left(\alpha^{\sigma(1-c)}\right) \tau_{l}$ for $\alpha \in R_{(\Xi)}^{\times}=T_{M}\left(\mathbb{Z}_{(\Xi)}\right)$, and $\widehat{\rho}\left(R_{(\Xi)}^{\times}\right)$leaves stable $\widehat{S}_{\mathfrak{l}}^{\text {ord }}$ and fixes $x_{\mathfrak{l}} \in \widehat{S}_{\mathfrak{l}}^{\text {ord }}$. This implies by universality of $S h^{(\Xi)}$ that we have an endo-isogeny $\widetilde{\theta}_{\mathfrak{L}}(\alpha) \in \operatorname{End}_{F}^{0}\left(A_{\mathfrak{L}}\right)$ for each $\alpha \in R_{(\Xi)}^{\times}$ such that $\widetilde{\theta}_{\mathfrak{L}}(\alpha) \circ \eta_{\mathfrak{L}}^{(\Xi)}=\eta_{\mathfrak{L}}^{(\Xi)} \circ \widehat{\rho}(\alpha)$ and the isogeny action of $\widetilde{\theta}_{\mathfrak{L}}(\alpha)$ is given by $t_{\sigma, \mathfrak{l}} \mapsto t_{\sigma, \mathfrak{l}}^{\alpha^{\sigma(1-c)}}$ for the l-adic Serre-Tate $\sigma$-coordinate $t_{\sigma, \mathfrak{l}}$. In other words, the triple $\left(E=M, \alpha_{q}=\left.\widetilde{\theta}_{\mathfrak{L}}(\alpha)\right|_{A_{\mathfrak{L}}\left[q^{\infty}\right]}, \widehat{\rho}\right)$ satisfies the assumption of Lemma 4.1 (3), and hence, by the $O$-linear embedding $\tilde{\theta}_{\mathfrak{L}}: M \hookrightarrow \operatorname{End}_{F}^{0}\left(A_{\mathfrak{L} / \mathbb{F}_{\mathfrak{L}}}\right)$, we have $M \hookrightarrow M^{\prime}$, which implies $\widetilde{\theta}_{\mathfrak{L}}: M \cong M^{\prime}$, since $M^{\prime}=\operatorname{End}_{F}^{0}\left(A_{\mathfrak{L}}\right)$ is a quadratic extension of F.

\section{LOCAL INDECOMPOSABILITY OF AN AVRM}

Let $(A, \lambda, \theta) / \mathcal{W}_{p}$ be an AVRM by multiplication by $O$ with good reduction. Thus the triple $(A, \lambda, \theta)$ is defined over a number field $k$ in which $p$ is unramified. We suppose that $\Sigma^{\text {ord }}:=\Sigma_{p}^{\text {ord }}$ is non-empty (so, $A_{0}=A \otimes_{\mathcal{W}} \overline{\mathbb{F}}_{p}$ is at least partially ordinary). By the Serre-Tate theory, we have $\widehat{S}^{\text {ord }} \cong \widehat{\mathbb{G}}_{m} \otimes_{\mathbb{Z}_{p}} O_{\Sigma^{\text {ord }}}$. We fix an isomorphism $\eta_{\mathfrak{p}}: \mu_{p^{\infty}} \otimes_{\mathbb{Z}_{p}} O_{\mathfrak{p}} \cong A\left[\mathfrak{p}^{\infty}\right]$ to fix the identification $\widehat{S}_{\mathfrak{p}}^{\text {ord }} \cong \widehat{\mathbb{G}}_{m} \otimes_{\mathbb{Z}_{p}} O_{\mathfrak{p}}$ for $\mathfrak{p} \in \Sigma_{p}^{\text {ord }}$. For a finite set of primes $\Xi$ including $p$ (which will be specified later), we will suitably choose an isomorphism $\eta^{(\Xi)}:\left(F_{\mathbb{A}}^{(\Xi), \infty}\right)^{2} \cong T^{(\Xi)} A \otimes_{\mathbb{Z}} \mathbb{Z}_{(\Xi)}$ defined over $\mathcal{W}_{\Xi}$ in the proof of the following theorem. Since $p$ is unramified in $k$ and $p \in \Xi$, by extending scalars to a finite extension of $k$ in the fraction field of $\mathcal{W}_{\Xi}$ if necessary, for a neat open compact subgroup $K \subset G\left(\mathbb{A}^{(\Xi), \infty}\right)$, we may assume that $\left(A, \bar{\lambda}, \eta^{(\Xi)}\right)$ (resp. $\left.\left(A, \bar{\lambda}, \bar{\eta}^{(\Xi)}\right)\right)$ gives rise to a point $x \in V\left(\mathcal{W}_{\Xi}\right)$ (resp. $\left.x \in V_{K}\left(\mathcal{W}_{k}\right)\right)$ for a geometrically irreducible component $V$ of $S h^{(\Xi)}$. Let $M=$ $\operatorname{End}_{F}^{0}\left(A_{0}\right)$, which is a CM field (see Lemma 2.2). Define $\widehat{\rho}: T_{M}\left(\mathbb{Z}_{(\Xi)}\right) \rightarrow G(\mathbb{A}(\Xi), \infty)$ by $\widetilde{\theta}_{\mathfrak{P}}(\alpha) \circ \eta_{0}^{(\Xi)}=\eta_{0}^{(\Xi)} \circ \widehat{\rho}(\alpha)$, where $\left(A_{0}, \lambda_{0}, \theta_{0}, \eta_{0}^{(\Xi)}\right)=\left(A, \lambda, \theta, \eta^{(\Xi)}\right) \times \mathcal{W}_{p} \overline{\mathbb{F}}_{p}$ and $\widetilde{\theta}_{\mathfrak{P}}$ is an extension of $\theta_{0}$ to $M$. Then $\widehat{\rho}(\alpha)$ acts on $\widehat{S}^{\text {ord }}$ by $t_{\mathfrak{p}} \mapsto t_{\mathfrak{p}}^{\alpha_{\mathfrak{p}}^{1-c}}$ for an embedding $M \ni \alpha \mapsto \alpha_{\mathfrak{p}} \in F_{\mathfrak{p}}$ and on $V$ through $\overline{\mathcal{E}}^{(\Xi)}$, where $c$ is the generator of $\operatorname{Gal}(M / F)$. The embedding $\alpha \mapsto \alpha_{\mathfrak{p}}$ induces $M_{\mathcal{P}}=F_{\mathfrak{p}}$ for a prime $\mathcal{P}$ of $M$ over $\mathfrak{p}$, and $A_{0}[\mathcal{P}]$ is connected. We write $\sigma_{\mathfrak{p}}: F_{\mathfrak{p}} \rightarrow \mathbb{C}_{p}$ for the embedding induced by the $\sigma$-projection of $W_{p} \otimes_{\mathbb{Z}} O=W_{p}^{I}$ to $W_{p} \subset \mathbb{C}_{p}$. If the place $\mathfrak{p}$ is associated to $i_{p} \circ \sigma$, we write $t_{\sigma}=\sigma_{\mathfrak{p}} \circ t_{\mathfrak{p}}$ and $\tau=\tau_{\sigma, p}=\log _{p} \circ t_{\sigma}$; thus, $\tau \circ \widehat{\rho}(\alpha)=i_{p}\left(\alpha^{\sigma(1-c)}\right) \tau$. By definition, if 
$i_{p} \circ \sigma$ gives rise to the $p$-adic place $\mathfrak{p} \in \Sigma_{p}^{o r d}, t_{\sigma}(A)=1(\Leftrightarrow \tau(A)=0)$ is equivalent to semi-simplicity of the $\mathfrak{p}$-adic Tate module $T_{\mathfrak{p}} A$ as an $I_{p}$-module for the inertia group $I_{p}$ in $\operatorname{Gal}(\overline{\mathbb{Q}} / \mathbb{Q})$ corresponding to $i_{p} \circ \sigma$. We want to prove

Theorem 5.1. Let the notation be as above. Suppose that $O$ is unramified at $p$ and that the place of $k$ induced by $i_{p}$ is unramified over $\mathbb{Q}$. If $A_{/ \overline{\mathbb{Q}}}=A \times_{\mathcal{W}} \overline{\mathbb{Q}}$ does not have complex multiplication, $T_{\mathfrak{p}} A$ is indecomposable as an $I_{p}$-module for each $\mathfrak{p} \in \Sigma_{p}^{o r d}$.

Proof. Contrary to the conclusion of the theorem, we assume that $T_{\mathfrak{p}} A$ for $\mathfrak{p} \in \Sigma_{p}^{\text {ord }}$ is a semi-simple $I_{p}$-module (so, $\left.t_{\mathfrak{p}}(A)=1\right)$. As before, $i_{p} \circ \sigma$ induces the place $\mathfrak{p}$ for $\sigma \in I$. Note that $A$ is defined over a number field $k \subset \overline{\mathbb{Q}}$ unramified at $p$ as $A$ is defined over unramified $\mathcal{W}$ and $A$ is projective. If $p$ is inert in $F$, then $t_{\sigma}(A)=1$ implies $t_{p}(A)=1$; thus, $A$ is the canonical lift of $A_{0}$ and so has complex multiplication, a contradiction. Thus we may assume that $F$ has at least two primes over $p$, though we do not use this fact in the rest of the proof.

We write primes of $F$ by lowercase Gothic letters and primes of $k$ by uppercase Gothic letters. The corresponding Roman character is the rational prime below. Pick a prime $\mathfrak{q}$ of $O$. Identify $T_{\mathfrak{q}} A \cong O_{\mathfrak{q}}^{2}$ and write $r_{\mathfrak{q}}: \operatorname{Gal}(\overline{\mathbb{Q}} / k) \rightarrow G L_{2}\left(O_{\mathfrak{q}}\right)$ for the Galois representation realized on $T_{\mathfrak{q}} A$.

Since $A$ is isotypic, as is well known (i.e., $A$ is isogenous to a product of copies of an absolutely simple AVRM over a finite extension of $k$; see GME], §5.3.1), we may assume that $A$ is absolutely simple. Then $D:=\operatorname{End}^{0}\left(A_{/ k}\right)$ is a (possibly commutative) division central simple algebra over a totally real field or a CM field $Z$ with integer ring $\mathfrak{r}$ (as $A \otimes_{k} \overline{\mathbb{Q}}$ does not have complex multiplication; see [GME], Lemma 5.3.2). Since $F$ is totally real, $Z \subset F$ has to be totally real. If $D$ is commutative, $D=F=Z$ is a totally real field, and if $D$ is not commutative, it is a division quaternion algebra over $Z$. By a theorem of Faltings (a solution of Tate's conjecture; see ARG], II), for $\operatorname{End}_{Z_{\mathfrak{q}}}^{0}\left(T_{\mathfrak{q}} A\right):=\operatorname{End}_{\mathfrak{r}_{\mathfrak{q}}}\left(T_{\mathfrak{q}} A\right) \otimes_{\mathfrak{r}_{\mathfrak{q}}} Z_{\mathfrak{q}}$, the algebra $Z_{\mathfrak{q}}\left[r_{\mathfrak{q}}\left(\operatorname{Gal}\left(\overline{\mathbb{Q}} / k^{\prime}\right)\right)\right] \subset \operatorname{End}_{Z_{\mathfrak{q}}}^{0}\left(T_{\mathfrak{q}} A\right)$ generated by $r_{\mathfrak{q}}\left(\operatorname{Gal}\left(\overline{\mathbb{Q}} / k^{\prime}\right)\right)$ over $Z_{\mathfrak{q}}$ is the commutant of $\operatorname{End}\left(A_{/ k}\right) \otimes_{\mathbb{Z}} \mathbb{Z}_{\mathfrak{q}}$ for any finite extension $k^{\prime} / k$, where $T_{\mathfrak{q}} A=$ $\lim _{n} A[\mathfrak{q}]^{n}$ and $Z_{\mathfrak{q}}$ is the $\mathfrak{q}$-adic completion of $Z \subset F$ (i.e., the closure of $Z$ in $\left.F_{\mathfrak{q}}\right)$. Let $C_{\mathfrak{q}}:=Z_{\mathfrak{q}}\left[r_{\mathfrak{q}}\left(\operatorname{Gal}\left(\overline{\mathbb{Q}} / k^{\prime}\right)\right)\right] \subset \operatorname{End}_{Z_{\mathfrak{q}}}^{0}\left(T_{\mathfrak{q}} A\right)$. Then $C_{\mathfrak{q}}$ is the commutant of $D_{\mathfrak{q}}=D \otimes_{Z} Z_{\mathfrak{q}}$ in $\operatorname{End}_{Z_{\mathfrak{q}}}^{0}\left(T_{\mathfrak{q}} A\right)$. Hence $C_{\mathfrak{q}}$ has center $Z_{\mathfrak{q}}$ and is independent of the choice of $k^{\prime}$. In particular, $C_{\mathfrak{q}} \otimes_{Z_{\mathfrak{q}}} D_{\mathfrak{q}} \cong \operatorname{End}_{Z_{\mathfrak{q}}}^{0}\left(T_{\mathfrak{q}} A\right) \cong M_{n}\left(Z_{\mathfrak{q}}\right)$ (an $n \times n$ matrix algebra) for a suitable $n$ (cf. BAL, VIII.10.2). This implies that the Brauer classes over $Z_{\mathfrak{q}}$ of $C_{\mathfrak{q}}$ and $D_{\mathfrak{q}}$ are inverses of each other (cf. BAL, VIII.10.4). Since $D$ is either a quaternion algebra over $Z$ or $D=F=Z$, the Brauer class of $D_{\mathfrak{q}}$ has order at most 2 in the Brauer group over $Z_{\mathfrak{q}}$. Thus $C_{\mathfrak{q}}$ is either isomorphic to a division quaternion algebra over $Z_{\mathfrak{q}}$ or $M_{2}\left(F_{\mathfrak{q}}\right)=M_{2}\left(Z_{\mathfrak{q}}\right)$.

Set $r=r_{\mathfrak{p}}($ so $\mathfrak{q}=\mathfrak{p})$. Since $M \ni \alpha \mapsto \alpha^{\sigma(1-c)} \in F_{\mathfrak{p}}$ implies $M_{\mathfrak{p}}=F_{\mathfrak{p}} \oplus F_{\mathfrak{p}} \hookrightarrow C_{\mathfrak{p}}$ (i.e., $\mathfrak{p}$ splits in $M)$, so, $C_{\mathfrak{p}} \cong M_{2}\left(F_{\mathfrak{p}}\right)$. Thus $\operatorname{Im}(r)$ contains an open subgroup of $S L_{2}\left(\mathbb{Z}_{q}\right) \subset C_{\mathfrak{q}}^{\times}$. This open image property follows from Ribet's argument in [Ri], IV. Indeed, assuming that $\operatorname{End}^{0}\left(A \otimes_{k} \overline{\mathbb{Q}}\right)=F($ an absolutely simple AVRM but a non-CM) and that $A$ has a place of multiplicative reduction, Ribet computed the Lie algebra of $\operatorname{Im}(r)$ and showed that the Lie algebra contains $\mathfrak{s l}_{2}\left(\mathbb{Z}_{p}\right)$ (which implies the open image property by exponentiating the Lie algebra). His assumptions are used to show that $C_{\mathfrak{p}}=M_{2}\left(F_{\mathfrak{p}}\right)$, and, using Faltings' result, Ribet's argument still 
works, and we get the open image property (see [BGK], Theorem C for more details of this point).

Since there are at least two non-isomorphic ramified semi-simple quadratic extensions of $\mathbb{Q}_{p}$, we have at least four non-isomorphic maximal tori in $S L(2) / \mathbb{Q}_{p}$ defined over $\mathbb{Q}_{p}$ having non-isomorphic projection to $P G L(2)$. Thus we can find a torus $T \subset G L(2)$ defined over $O_{\mathfrak{p}}$ such that

(p1) $T\left(O_{\mathfrak{p}}\right) \cap S L_{2}\left(\mathbb{Z}_{p}\right)$ is an anisotropic maximal torus (i.e., $T\left(O_{\mathfrak{p}}\right)$ is isomorphic to the norm 1 subgroup of the multiplicative group of a field extension of $\left.F_{\mathfrak{p}}\right)$,

(p2) $T\left(O_{\mathfrak{p}}\right)$ remains an anisotropic maximal torus (i.e., for example, if $T\left(O_{\mathfrak{p}}\right) \cap$ $S L_{2}\left(\mathbb{Z}_{p}\right)$ comes from a ramified quadratic extension of $\mathbb{Q}_{p}, T\left(O_{\mathfrak{p}}\right)$ remains anisotropic as $F_{\mathfrak{p}} / \mathbb{Q}_{p}$ is unramified).

We pick $\alpha \in T\left(O_{\mathfrak{p}}\right) \cap \operatorname{Im}(r) \cap S L_{2}\left(\mathbb{Z}_{p}\right)$ so that $T$ is the centralizer of $\alpha$ (i.e., $\alpha$ has two distinct eigenvalues in $\mathbb{C}_{p}$ ). Since the isomorphism class of the centralizer of $\alpha$ (as a tori over $O_{\mathfrak{p}}$ ) is determined by $\alpha \bmod p^{j}$ for sufficiently large $j$, by Chebotarev density, changing $T$ in its isomorphism/conjugacy class if necessary, we may choose a prime $\mathfrak{L}$ outside $p$ so that

- $A$ has good reduction at $\mathfrak{L}$,

- $r\left(F_{r o b}\right)$ commutes with $\alpha$ (i.e., $\left.r\left(F_{\mathfrak{L} o b_{\mathfrak{L}}}\right) \in T\left(F_{\mathfrak{p}}\right)\right)$.

For the eigenvalues $\lambda$ of $r\left(F r o b_{\mathfrak{L}}\right)$, the quadratic extension $M^{\prime}=F[\lambda]$ is nonisomorphic to $M=\operatorname{End}_{F}^{0}\left(A_{0 / \overline{\mathbb{F}}_{p}}\right)$ over $F$ (i.e., $\mathfrak{p}$ is non-split in $M^{\prime}$, but $\mathfrak{p}$ splits in $M)$. Since $A$ has $\Sigma_{l}^{\text {ord }} \neq \emptyset$ for primes $\mathfrak{L}$ of $k$ of Dirichlet density 1 (after replacing $k$ by its finite extension if necessary; see Section 7 in the text and [O], 2.7 and [N], Introduction), we may assume that $\Sigma_{l}^{o r d}$ contains a prime induced by $i_{l} \circ \sigma$ (i.e., we may assume that $\mathfrak{l}$ splits in $\left.M^{\prime}\right)$; thus, we have $\operatorname{End}_{F}^{0}\left(A \otimes \mathcal{W}_{l} \overline{\mathbb{F}}_{l}\right)=M^{\prime}$.

Let $\Xi=\{p, l\}$ for the residual characteristic $l$ of $\mathfrak{l}$. Then $A_{0}$ has complex multiplication by a CM field $M_{/ F}$. Thus $A_{0}\left[q^{\infty}\right] \cong\left(F_{q} / O_{q}\right)^{2}$ over $\overline{\mathbb{F}}_{p}$ for any prime $q \notin \Xi$. Since $A_{0}$ is defined over $\mathbb{F}_{\mathfrak{P}}$, it has the Frobenius endomorphism $\phi$ over $\mathbb{F}_{\mathfrak{P}}$ and $M=F[\phi]=\operatorname{End}_{F}^{0}\left(A_{0 / \mathbb{F}_{\mathfrak{P}}}\right)$ is a CM quadratic extension of $F$ (by Lemma 2.2). Thus we can find an $R$-linear isomorphism $\eta_{0}^{(\Xi)}: \widehat{R}^{(\Xi)} \cong T^{(\Xi)} A_{0}$ for $R=O[\phi]$, where $\widehat{R}=\prod_{q} R_{q}$ and $\widehat{R}^{(\Xi)}=\prod_{q \notin \Xi} R_{q}$. Thus, identifying $\widehat{R}^{(\Xi)} \otimes_{\mathbb{Z}} \mathbb{Q}=\left(F_{\mathbb{A}}^{(\Xi), \infty}\right)^{2}$, we may define an embedding $\widehat{\rho}: M^{\times} \hookrightarrow G\left(\mathbb{A}^{(\Xi), \infty}\right)$ by $\widetilde{\theta}_{\mathfrak{P}}(\alpha) \circ \eta_{0}^{(\Xi)}=\eta_{0}^{(\Xi)} \circ \widehat{\rho}(\alpha)$. Since $A\left[q^{\infty}\right]$ for $q \notin \Xi$ is étale over $\mathcal{W}_{\Xi}$, this level structure $\eta_{0}^{(\Xi)}$ lifts uniquely to a level structure $\eta^{(\Xi)}$ of $A$. Then the point $x_{0} \in S h^{(\Xi)}\left(\overline{\mathbb{F}}_{p}\right)$ carrying $\left(A_{0}, \lambda_{0}, \eta_{0}^{(\Xi)}\right)$ is fixed by $\widehat{\rho}\left(T_{M}\left(\mathbb{Z}_{(\Xi)}\right)\right)$ by Lemma 4.1 .

Suppose that $\left.r_{\mathfrak{p}}\right|_{I_{p}}$ is semi-simple for $\mathfrak{p} \in \Sigma^{\text {ord }}$ for $\mathfrak{p}$ induced by $\sigma: F \rightarrow \overline{\mathbb{Q}}_{p}$. Thus by semi-simplicity, the following connected-étale exact sequence

$$
0 \rightarrow A\left[\mathfrak{p}^{\infty}\right]^{\circ}\left(\overline{\mathbb{Q}}_{p}\right) \rightarrow A\left[\mathfrak{p}^{\infty}\right]\left(\overline{\mathbb{Q}}_{p}\right) \rightarrow A\left[\mathfrak{p}^{\infty}\right]^{e t}\left(\overline{\mathbb{Q}}_{p}\right) \rightarrow 0
$$

of $I_{p}$-modules splits, and hence the connected-étale sequence of Barsotti-Tate $O$ modules

$$
0 \rightarrow A\left[\mathfrak{p}^{\infty}\right]_{/ W_{p}}^{\circ} \rightarrow A\left[\mathfrak{p}^{\infty}\right]_{/ W_{p}} \rightarrow A\left[\mathfrak{p}^{\infty}\right]_{/ W_{p}}^{e t} \rightarrow 0
$$

also splits (cf. [T] ). In particular, we have $\mathfrak{p}=\mathcal{P} \overline{\mathcal{P}}$ for prime ideals $\mathcal{P} \neq \overline{\mathcal{P}}$ in $R=\operatorname{End}_{O}\left(A_{0 / \mathbb{F}_{p}}\right) \subset M$ so that $A_{0}\left[\mathfrak{p}^{\infty}\right]^{\circ}=A_{0}\left[\mathcal{P}^{\infty}\right]$ and $A_{0}\left[\mathfrak{p}^{\infty}\right]^{e t}=A_{0}\left[\overline{\mathcal{P}}^{\infty}\right]$. The isomorphism $\eta_{\mathfrak{p}}: \mu_{p^{\infty}} \otimes_{\mathbb{Z}_{p}} O_{\mathfrak{p}} \cong A\left[\mathfrak{p}^{\infty}\right]^{\circ}$ is defined over $\mathcal{W}_{p}$. Recall $M^{\times} \ni \alpha \mapsto \alpha_{\mathfrak{p}} \in$ 
$F_{\mathfrak{p}}$ given by $\widetilde{\theta}_{\mathfrak{P}}(\alpha) \circ \eta_{\mathfrak{p}}=\eta_{\mathfrak{p}} \circ \alpha_{\mathfrak{p}}$. By definition, we also have $t_{\mathfrak{p}}(A)=1$; therefore, $\tau=\log _{p} \circ \sigma \circ t_{\mathfrak{p}}$ satisfies $\tau \circ \widehat{\rho}(\alpha)=i_{p}\left(\alpha^{\sigma(1-c)}\right) \tau$ for an extension $\sigma: M \hookrightarrow \overline{\mathbb{Q}}_{p}$ of $\sigma$. Thus by Theorem 4.5, the corresponding coordinate $\tau_{l}=\tau_{\sigma, l}$ on the l-adic deformation space satisfies the same invariance property (i.e., they are a $\hat{\rho}$-eigen coordinate and $\left.\tau_{l}(A)=0\right)$. By our choice, $M^{\prime}=\operatorname{End}_{F}^{0}\left(A \otimes \mathcal{W}_{l} \overline{\mathbb{F}}_{l}\right)$ with $M \nsubseteq M^{\prime}$, which is a contradiction as $M^{\prime}=\operatorname{End}_{F}^{0}\left(A \otimes \mathcal{W}_{l} \overline{\mathbb{F}}_{l}\right) \cong \operatorname{End}_{F}^{0}\left(A_{0 / \overline{\mathbb{F}}_{p}}\right)=M$ (as $F$ algebras) by Corollary 4.6 .

\section{CM PERIODS AND PROPORTIONALITY CONSTANTS}

In the proof of the indecomposability in the previous section, proportionality of $\tau_{\mathfrak{p}}$ and $\tau_{\mathfrak{l}}$ proven in Theorem 4.5 played an important role. We study the proportionality constant of our $\widehat{\rho}$-eigen $\varphi$-coordinate at each $v \in \Xi$ in terms of CM periods of Katz and Shimura, starting with a CM abelian variety (not with the non-CM abelian variety as we did so far). We show that the constant is the ratio of the square of $v$-adic CM periods. This section is independent of the proof of the local indecomposability (and the reader with interest only in local indecomposability may skip this section).

Let $x_{0} \in S h^{(\Xi)}\left(\overline{\mathbb{F}}_{p}\right)$ be in the ordinary locus. The Serre-Tate deformation space of $x_{0}$ is then isomorphic to $\widehat{S}_{p}=\widehat{S}_{p}^{\text {ord }}=\widehat{\mathbb{G}}_{m} \otimes_{\mathbb{Z}} O$ (as $p$ is assumed to be unramified in $\left.F_{/ \mathbb{Q}}\right)$ embedded into $S h_{/ W_{p}}^{(\Xi)}$. Let $x_{1} \in S h^{(\Xi)}$ be the origin $\mathbf{1} \in \widehat{S}_{p} \hookrightarrow S h_{/ W_{p}}^{(\Xi)}$, and let $\left(A_{1}, \bar{\lambda}_{1}, \theta_{1}, \eta_{1}^{(\Xi)}\right) / \mathcal{W}_{k}$ be the CM abelian variety (the canonical lift) sitting over $x_{1}$ (which is originally defined over $W_{p}$ but it descends to $\mathcal{W}_{k}$ for a number field $k$ by the theory of complex multiplication [ACM] $)$, where $\theta_{1}: M \cong \operatorname{End}_{F}^{0}\left(A_{1 / \mathcal{W}_{\Xi}}\right)$. This is the CM point we study. Suppose that $A_{1} \times \mathcal{W}_{k} \overline{\mathbb{F}}_{l}$ is also ordinary for $l \in \Xi$ different from $p$. So we may assume that $\Xi=\{p, l\}$, and we suppose that $l$ is unramified in $F_{/ \mathbb{Q}}$.

At $v=p, l$, we fix $\eta_{v}: \mu_{v^{\infty}} \otimes_{\mathbb{Z}} O \hookrightarrow A_{1}\left[v^{\infty}\right]$ defined over $\mathcal{W}_{v}$. This is possible as $\mathcal{W}_{v}$ is a strict henselization of $\mathbb{Z}_{(v)}$. This level structure (together with $\eta_{1}^{(\Xi)}$ ) gives rise to a point on the $v$-adic Igusa tower

$$
I g_{v, \infty}:=\operatorname{Isom}_{\mathcal{W}_{v}}\left(\mu_{v} \otimes_{\mathbb{Z}} O_{/ S h_{\infty}^{\text {ord }}}, \mathbf{A}_{/ S h_{\infty}^{\text {ord }}}\left[v^{\infty}\right]^{\circ}\right)
$$

for the universal abelian scheme $\mathbf{A}$ over the ordinary locus $S h_{\infty}^{\text {ord }}$ of $S h^{(v)}$. We again simply write $x_{1}$ for this point on $I g_{v, \infty}$. By fixing $\eta_{v}$, we have a unique identification of $\widehat{S}_{v}$ with $\widehat{\mathbb{G}}_{m} \otimes_{\mathbb{Z}} O$. Define $\widehat{\rho}: T_{M}\left(\mathbb{Z}_{(\Xi)}\right) \rightarrow G(\mathbb{A}(\Xi), \infty)$ by $\theta_{1}(\alpha) \circ \eta_{1}^{(\Xi)}=\eta_{1}^{(\Xi)} \circ \widehat{\rho}(\alpha)$. Then write $\tau_{v, \varphi}$ for the $\widehat{\rho}$-eigen $\varphi$-coordinate of $\left(\widehat{S}_{v}\right)^{v \text {-an }}$ if $v=p, l$. In other words, if $v=p$ for the $\varphi$-projection $\varphi_{\mathfrak{p}}: W_{p} \otimes_{\mathbb{Z}} O \rightarrow W_{p}$, we have $\tau_{p, \varphi}=\log _{p} \circ \varphi_{\mathfrak{p}} \circ t_{\mathfrak{p}_{\varphi}}$ for the Serre-Tate coordinate $t_{\mathfrak{p}_{\varphi}}$ of $\widehat{S}_{\mathfrak{p}_{\varphi}}^{\text {ord }}=\widehat{\mathbb{G}}_{m} \otimes_{\mathbb{Z}_{p}} O_{\mathfrak{p}_{\varphi}}$.

By ordinarity, any prime factor $\mathfrak{v} \mid v$ (for $v=p, l$ ) in $O$ splits in $M$; in particular, $v$ is unramified in $M_{/ \mathbb{Q}}$. Write $\Phi$ for the CM type of $A_{1}$; thus, $\Phi$ is a collection of half of the embeddings of $M$ into $\overline{\mathbb{Q}}$ such that $\sum_{\phi \in \Phi} \phi$ as a representation of $M$ is isomorphic to $\Omega_{A_{1} / k} \otimes_{k} \overline{\mathbb{Q}}$. Supposing that $k$ is large containing all conjugates of $M$ in $\overline{\mathbb{Q}}$, we decompose $H^{0}\left(A_{1}, \Omega_{A_{1} / \mathcal{W}_{k}}\right)=\bigoplus_{\phi \in \Phi} \mathcal{W}_{k} \omega_{\phi}$ with nowhere vanishing differentials $\omega_{\phi}$ having an eigenproperty $\theta_{1}(\alpha)^{*} \omega_{\phi}=\phi(\alpha) \omega_{\phi}$ for $\alpha \in M=\operatorname{End}_{F}^{0}\left(A_{1}\right)$. As is well known, $R:=\theta_{1}^{-1}\left(\operatorname{End}_{O}\left(A_{1 / \mathcal{W}_{k}}\right)\right)$ is an $O$-order of $M$, in which any prime $\mathfrak{v} \mid v$ of $R$ splits (see Lemma 2.2). 
Fixing complex uniformization $L \hookrightarrow \mathbb{C}^{\Phi} \rightarrow A_{1}(\mathbb{C})$ for a proper $R$-ideal $L \subset R$. Writing the variable of $\mathbb{C}^{\Phi}$ as $u=\left(u_{\phi}\right), \omega_{\infty, \phi}:=d u_{\phi} \in H^{0}\left(A_{1}, \Omega_{A_{1} / \mathbb{C}}\right)$ satisfies $\theta_{1}(\alpha)^{*} \omega_{\infty, \phi}=\phi(\alpha) \omega_{\infty, \phi}$ for $\alpha \in M$. Thus $\omega_{\phi}$ and $\omega_{\infty, \phi}$ are proportional, getting Shimura's CM period $\Omega_{\infty, \phi} \in \mathbb{C}^{\times}$by $\omega_{\phi}=\Omega_{\infty, \phi} \omega_{\infty, \phi}$. Since $\int_{\gamma} \omega_{\infty, \phi} \in \phi(L) \subset \mathcal{W}_{k}$ for all $\gamma \in \pi_{1}\left(A_{1}(\mathbb{C}), \mathbf{0}\right)$ for the origin $\mathbf{0} \in A_{1}$, we have the classical algebraic period identity $\int_{\gamma} \omega_{\phi} \in \mathcal{W} \cdot \Omega_{\infty, \phi}$. We put $\Omega_{\infty}=\left(\Omega_{\infty, \phi}\right)_{\phi \in \Phi} \in\left(\mathbb{C}^{\times}\right)^{\Phi}$.

For $v=p, l$, the level structure $\eta_{v}$ induces $\widehat{\eta}_{v}: \widehat{\mathbb{G}}_{m} \otimes_{\mathbb{Z}} O=\varliminf_{n}\left(\mu_{v^{n}} \otimes_{\mathbb{Z}} O\right) \cong \widehat{A}_{1}$ defined over $W_{v}$ for the formal completion $\widehat{A}_{1}$ of $A_{1}$ along the origin $\mathbf{0} \in A_{1}\left(\overline{\mathbb{F}}_{v}\right)$. Thus, writing $\mathbb{G}_{m}=\operatorname{Spec}\left(W\left[t, t^{-1}\right]\right)$, the push forward $\widehat{\eta}_{v, *}\left(\frac{d t}{t} \otimes 1\right)$ can be written as a unique sum $\widehat{\eta}_{v, *}\left(\frac{d t}{t} \otimes 1\right)=\sum_{\phi \in \Phi} \omega_{v, \phi}$ satisfying $\theta_{1}(\alpha)^{*} \omega_{v, \phi}=\phi(\alpha) \omega_{v, \phi}$ for $\alpha \in M=\operatorname{End}_{F}^{0}\left(A_{1}\right)$. We then define Katz's $v$-adic period $\Omega_{v, \phi}$ by $\omega_{\phi}=\Omega_{v, \phi} \omega_{v, \phi}$. We put $\Omega_{v}=\left(\Omega_{v, \phi}\right)_{\phi \in \Phi} \in W_{v}^{\Phi}$ (see [K1] ). Thus we get

Proposition 6.1. Under our identification $\mathbb{C}_{p}=\mathbb{C}$, the proportionality constant of $\omega_{p, \phi}$ and $\omega_{\infty, \phi}$ both in $H^{0}\left(A_{1}, \Omega_{A_{1} / \mathbb{C}}\right)=H^{0}\left(A_{1}, \Omega_{A_{1} / \mathbb{C}_{p}}\right)$ is given by $\Omega_{\infty, \phi} / \Omega_{p, \phi}$ for each $\phi \in \Phi$. In other words, we have

$$
\Omega_{p, \phi} \omega_{p, \phi}=\omega_{\phi}=\Omega_{\infty, \phi} \omega_{\infty, \phi}=\Omega_{\infty, \phi} d u_{\phi}
$$

for $\omega_{\phi} \in H^{0}\left(A_{1}, \Omega_{A_{1} / \mathcal{W} \Xi}\right)$.

Assuming that $A_{1}$ is also ordinary at $l \in \Xi$, applying the above proposition to $\{p, \infty\}$ and then to $\{l, \infty\}$, we get

Corollary 6.2. Under our identification $\mathbb{C}_{p}=\mathbb{C}_{l}$, the proportionality constant of $\omega_{p, \phi}$ and $\omega_{l, \phi}$ both in $H^{0}\left(A_{1}, \Omega_{A_{1} / \mathbb{C}_{p}}\right)=H^{0}\left(A_{1}, \Omega_{A_{1} / \mathbb{C}_{l}}\right)$ is given by $\Omega_{l, \phi} / \Omega_{p, \phi}$ for each $\phi \in \Phi$. In other words, we have

$$
\Omega_{p, \phi} \omega_{p, \phi}=\omega_{\phi}=\Omega_{l, \phi} \omega_{l, \phi}
$$

for $\omega_{\phi} \in H^{0}\left(A_{1}, \Omega_{A_{1} / \mathcal{W} \Xi}\right)$.

We have three Kodaira-Spencer maps:

$$
\begin{aligned}
& \left(\pi_{*} \Omega_{\mathbf{A} / S h_{/ \mathcal{W}_{k}}^{(p)}}[\varphi]\right)^{\otimes 2}=\underline{\omega}^{2 \varphi} \mathcal{W}_{k} \cong \Omega_{S h^{(p)} / \mathcal{W}_{k}}[\varphi], \\
& \left(\pi_{*} \Omega_{\mathbf{A} / \widehat{S}_{p / W_{p}}^{o r d}}[\varphi]\right)^{\otimes 2}=\underline{\omega}_{/ W_{p}}^{2 \varphi} \cong \Omega_{\widehat{S}_{p} / W_{p}}[\varphi], \\
& \left(\pi_{*} \Omega_{\mathbf{A} / \widehat{S}_{l / W_{l}}^{o r d}}[\varphi]\right)^{\otimes 2}=\underline{\omega}_{/ W_{l}}^{2 \varphi} \cong \Omega_{\widehat{S}_{l} / W_{l}}[\varphi] .
\end{aligned}
$$

Here A denotes the universal abelian scheme corresponding to the bases $S h_{\infty}^{\text {ord }}$, $\widehat{S}_{\mathfrak{p}}^{\text {ord }}$ and $\widehat{S}_{\mathfrak{l}}^{\text {ord }}$ whose origin corresponds to $x_{1}$. As before, "[$[\varphi]$ " indicates the $\varphi$ eigenspace under the natural action of $O$. Taking the fiber at $x_{1}$ of (6.1), we get

$$
\begin{aligned}
& \left(H^{0}\left(A_{1}, \Omega_{A_{1} / \mathcal{W}_{k}}[\varphi]\right)\right)^{\otimes 2}=\underline{\omega}^{2 \varphi}\left(x_{1}\right) / \mathcal{W}_{k} \cong \Omega_{S h^{(p)} / \mathcal{W}_{k}}[\varphi]\left(x_{1}\right), \\
& \left(H^{0}\left(A_{1}, \Omega_{A_{1} / W_{p}}[\varphi]\right)\right)^{\otimes 2}=\underline{\omega}^{2 \varphi}\left(x_{1}\right) / W_{p} \cong \Omega_{\widehat{S}_{p} / W_{p}}[\varphi]\left(x_{1}\right), \\
& \left(H^{0}\left(A_{1}, \Omega_{A_{1} / W_{l}}[\varphi]\right)\right)^{\otimes 2}=\underline{\omega}^{2 \varphi}\left(x_{1}\right) / W_{l} \cong \Omega_{\widehat{S}_{l} / W_{l}}[\varphi]\left(x_{1}\right) .
\end{aligned}
$$

We can identify $\Phi$ with $I$ by $\left.\phi \mapsto \phi\right|_{F}=: \varphi$. Then $\varphi$-eigenspace under the action of $O$ is identical to the $\phi$-eigenspace under the action of $R$. The data

$$
\left\{\omega_{\phi} \in H^{0}\left(A_{1}, \Omega_{A_{1} / \mathcal{W}_{k}}[\varphi]\right), \omega_{p, \phi} \in H^{0}\left(A_{1}, \Omega_{A_{1} / W_{p}}[\varphi]\right), \omega_{l, \phi} \in H^{0}\left(A_{1}, \Omega_{A_{1} / W_{l}}[\varphi]\right)\right\}
$$


give rise to the corresponding differentials

$$
\begin{aligned}
\left\{\omega_{\phi}^{\otimes 2}=\omega_{\phi} \otimes \omega_{\phi} \in \Omega_{\mathbf{A} / S h^{\prime} / \mathcal{W}_{k}}^{(p)}[\varphi]\left(x_{1}\right), \omega_{p, \phi}^{\otimes 2} \in\right. & \Omega_{\mathbf{A} / \widehat{S}_{p / W_{p}}}[\varphi]\left(x_{1}\right), \\
& \left.\omega_{l, \phi}^{\otimes 2} \in \Omega_{\mathbf{A} / \widehat{S}_{l / W_{l}}}[\varphi]\left(x_{1}\right)\right\} .
\end{aligned}
$$

Define $\widehat{\rho}: T_{M}\left(\mathbb{Z}_{(\Xi)}\right) \rightarrow G\left(\mathbb{A}^{(\Xi), \infty}\right)$ by $\theta_{1}(\alpha) \circ \eta_{1}^{(\Xi)}=\eta_{1}^{(\Xi)} \circ \widehat{\rho}(\alpha)$. Write $\tau_{v, \varphi}$ for the additive Serre-Tate $\widehat{\rho}$-eigen $\varphi$-coordinate of $\left(\widehat{S}_{p}\right)^{v \text {-an }}$ for $v=l, p$. Then we define the proportionality constant $C_{\varphi}^{v, w}$ to be $\tau_{v, \varphi}=C_{\varphi}^{v, w} \cdot \tau_{w, \varphi}$. The constant $0 \neq C_{\varphi}^{v, w}$ is determined by comparing $d \tau_{v, \varphi}$ and $d \tau_{w, \varphi}$ in $\Omega_{S h^{(p)} / \mathbb{C}_{v}}[\varphi]\left(x_{1}\right)=\Omega_{S h^{(p)} / \mathbb{C}_{w}}[\varphi]\left(x_{1}\right)$, in which we have algebraic $\omega_{\phi}^{\otimes 2}$. Note that by our construction and [K], Main Theorem 3.7.1, we have $d \tau_{v, \varphi}=\frac{d t_{v, \varphi}}{t_{v, \varphi}}=\omega_{v, \phi}^{\otimes 2}$ with $t_{v, \varphi}=\varphi \circ t_{\mathfrak{v}_{\varphi}}$ for the unique continuous extension $\varphi: F_{\mathfrak{v}_{\varphi}} \hookrightarrow \mathbb{C}_{v}$ of the original $\varphi: F \hookrightarrow \overline{\mathbb{Q}}$. Thus from Corollary 6.2, we get

Theorem 6.3. Under our identification $\mathbb{C}_{p}=\mathbb{C}_{l}$, the proportionality constant $C_{\varphi}^{p, l}$ of $\tau_{p, \varphi}$ and $\tau_{l, \varphi}$ is given by $\Omega_{l, \phi}^{2} / \Omega_{p, \phi}^{2}$ for each $\varphi \in I$, where $\phi \in \Phi$ corresponds to $\varphi$ by $\left.\phi\right|_{F}=\varphi$. In other words, we have

$$
\Omega_{p, \phi}^{2} d \tau_{p, \varphi}=\omega_{\phi}^{\otimes 2}=\Omega_{l, \phi}^{2} d \tau_{l, \varphi} \text { in } \Omega_{S h^{(\Xi)} / \mathbb{C}_{p}}\left(x_{1}\right)=\Omega_{S h^{(\Xi)} / \mathbb{C}_{l}}\left(x_{1}\right)
$$

for $\omega_{\phi}^{\otimes 2} \in \underline{\omega}^{2 \varphi}$.

Question 6.4. If we start with the non-CM $\operatorname{AVRM}(A, \bar{\lambda}, \theta)_{/ k}$, we can associate two $p$-adic "periods" $\tau_{p, \varphi}(A)$ for each $\varphi \in \Sigma_{p}^{o r d}$ and $\Omega(A)_{p, \varphi}$ given by $\Omega(A)_{p, \varphi} \omega_{p, \varphi}=\omega_{\varphi}$ choosing a generator $\omega_{\varphi}$ of $H^{0}\left(A, \Omega_{A} \mathcal{W}_{k}[\varphi]\right)$ over $\mathcal{W}_{k}$. It is an interesting question if these periods are related to some $p$-adic $L$-values coming out of the abelian scheme $A_{/ k}$.

If $A$ is an ordinary abelian variety with $\mathrm{CM}$, we have $\tau_{p, \varphi}(A)=0\left(\operatorname{as} t_{\mathfrak{p}}(A)=1\right)$. Thus, we get only one non-trivial period, the Katz CM period $\Omega(A)_{p, \varphi}$. By a result of Shimura, the value of an algebraic modular form $f$ at the CM abelian variety " $A$ " with complex invariant differentials $\left\{d u_{\phi}\right\}_{\phi}$ is equal to a monomial of $\left\{\Omega(A)_{\infty, \varphi}\right\}_{\varphi}$ up to an algebraic number which is the value of $f$ at $\left(A, \omega_{\phi}\right)_{\phi}$. Similarly, by a result of Katz, the value of $f$ at the $\mathrm{CM}$ abelian variety " $A$ " with $p$-adic invariant differentials $\left\{\omega_{p, \phi}\right\}$ of an algebraic modular form is equal to the same monomial of $\left\{\Omega(A)_{p, \varphi}\right\}_{\varphi}$ times $f\left(\left(A, \omega_{\phi}\right)_{\phi}\right)$. An elementary treatment of the theory of Katz and Shimura can be found in [EAI], Section 1.3. If $A$ is non-CM and has ordinary good reduction, $\tau_{p, \varphi}(A)$ plays a role similar to the Tate period of an abelian variety with multiplicative reduction, though as of yet we do not know much about its arithmetic meaning.

\section{Density of PARTIALly ORDINARY PRIMES}

For the reader's convenience, we give a sketch of a simple proof of the density result for partially ordinary primes used in the proof of Theorem 5.1. We consider the set $\mathcal{S}^{\text {ord }}$ of prime ideals of $k$ unramified over $\mathbb{Q}$ at which $A$ has partially ordinary good reduction for a given non-CM absolutely simple $\operatorname{AVRM}(A, \lambda, \theta)$. Here $(A, \lambda, \theta)$ is defined over the number field $k \subset \overline{\mathbb{Q}}$ and $A$ has partially ordinary good reduction at $\mathfrak{P}$ if $A$ extends to an abelian scheme over $\mathfrak{O}_{\mathfrak{P}}$ and $A[p]\left(\overline{\mathbb{F}}_{p}\right) \neq 0$ for an algebraic closure $\overline{\mathbb{F}}_{p}$ of $\mathfrak{O} / \mathfrak{P}$. First suppose that $k / \mathbb{Q}$ is a Galois extension. Then the set 
of primes of $k$ split over $\mathbb{Q}$ has Dirichlet density 1. Let $\mathcal{S}_{f}^{\text {ord }} \subset \mathcal{S}^{\text {ord }}$ be the set of all primes in $\mathcal{S}^{\text {ord }}$ with residual degree $f$. We have $\mathcal{S}^{\text {ord }}=\bigsqcup_{f=1}^{[k: \mathbb{Q}]} \mathcal{S}_{f}^{\text {ord }}$. If a prime $\mathfrak{P}$ in $\mathfrak{O}$ has residual degree 1 , the reduction $A_{\mathfrak{P}}=A \otimes_{\mathfrak{O}_{\mathfrak{P}}} \mathfrak{O} / \mathfrak{P}$ has the $p$-power Frobenius endomorphism $\phi$ whose eigenvalue $\alpha$ satisfies $\left|\alpha^{\varphi}\right|=\sqrt{p}$ for all $\varphi \in \operatorname{Gal}(\overline{\mathbb{Q}} / \mathbb{Q})$. Since $a=\alpha+\alpha^{c}$ is in $O$, for the complex conjugation $c$ (i.e., the Rosati involution of $\operatorname{End}_{O}\left(A_{\mathfrak{P}}\right)$ with respect to $\lambda$ ) (as $A_{\mathfrak{P}}$ is defined over $\left.\mathfrak{O} / \mathfrak{P}=\mathbb{F}_{p}\right)$, we get $\left|a^{\varphi}\right| \leq 2 \sqrt{p}$. Taking the norm to $\mathbb{Q}$, we have $\left|N_{F / \mathbb{Q}}(a)\right| \leq 2^{d} p^{d / 2}$. Thus if $p \mid(a)$ is in $\mathfrak{O}$, we have $p^{d} \mid N_{F / \mathbb{Q}}(a)$; therefore, $p^{d} \leq\left|N_{F / \mathbb{Q}}(a)\right| \leq 2^{d} p^{d / 2}$ as long as $a \neq 0$. Thus if $a \neq 0$, we have a prime $\mathfrak{p} \nmid a$ in $O$ above $p$ for $p \geq 5$. Then as is well known, this implies that $A[\mathfrak{p}]\left(\overline{\mathbb{F}}_{p}\right) \neq 0$ (i.e., $\mathfrak{p} \in \Sigma_{p}^{\text {ord }}$ ). For any $p$-adic Galois representation $\rho: \operatorname{Gal}(\overline{\mathbb{Q}} / k) \rightarrow G L_{m}\left(\mathbb{Q}_{p}\right)$, fix a Haar measure on $\operatorname{Im}(\rho)$ with volume 1. By Chebotarev density (e.g., ALR, I-8, Corollary 2 combined with the Exercise there and [Se, $\S 2.1$ ), the density of primes of $k$ whose Frobenius falling in a closed set of volume 0 is equal to 0 . Apply this to the Galois representation $\rho_{\mathfrak{r}}$ on the $\mathfrak{l}$-adic Tate module of $A_{/ k}$ taking a prime $\mathfrak{l}$ of $F$. By the result of [BGK], Theorem $\mathrm{C}$, the set of $\mathfrak{P}$ whose Frobenius has trace 0 has volume 0 . Thus for non-CM AVRM $A$, the set of primes $\mathfrak{P}$ with $a=0$ has Dirichlet density 0 by the quantitative Chebotarev density theorem (although Serre works over $\mathbb{Q}$, his argument generalizes to a number field $k$ without any modification). If $k / \mathbb{Q}$ is not a Galois extension, we just replace $k$ by its Galois closure and apply the above argument. Since as in Theorem 5.1 we say that $A$ does not have CM if $A \times{ }_{k} \overline{\mathbb{Q}}$ does not have $\mathrm{CM}$ (i.e., $\operatorname{End}_{F}^{0}\left(A \times_{k} \overline{\mathbb{Q}} / \overline{\mathbb{Q}}\right)=F$ ), being non-CM is insensitive to any base change finite over $k$. In particular, the abelian variety $A$ over the Galois closure of $k$ does not have CM. Thus we have

Proposition 7.1. Let $(A, \lambda, \theta)$ be a non-CM AVRM defined over $k \subset \overline{\mathbb{Q}}$. Replacing $k$ by its finite extension if necessary, the set $\mathcal{S}_{1}^{\text {ord }}$ for a given AVRM has Dirichlet density equal to 1.

It is interesting to know the Dirichlet density of the set $\left\{l \mid \mathfrak{L} \in \mathcal{S}_{f}^{\text {ord }}\right\}$ of rational primes if $f>1$ (this question is not about the density over $k$ but over $\mathbb{Q}$ ). The result [O], 2.7 tells us something about $\mathcal{S}_{2}^{\text {ord }}$.

\section{ACKNOWLEDGEMENT}

The author thanks Brian Conrad for some help in identifying an optimal reference in rigid analytic geometry for quotations used in this work.

\section{REFERENCES}

[ABV] David Mumford, Abelian varieties, Tata Institute of Fundamental Research Studies in Mathematics, vol. 5, Published for the Tata Institute of Fundamental Research, Bombay, 2008. With appendices by C. P. Ramanujam and Yuri Manin; Corrected reprint of the second (1974) edition. MR2514037 (2010e:14040)

[ACM] Goro Shimura, Abelian varieties with complex multiplication and modular functions, Princeton Mathematical Series, vol. 46, Princeton University Press, Princeton, NJ, 1998. MR 1492449 (99e:11076)

[ALR] Jean-Pierre Serre, Abelian l-adic representations and elliptic curves, Research Notes in Mathematics, vol. 7, A K Peters Ltd., Wellesley, MA, 1998. With the collaboration of Willem Kuyk and John Labute; Revised reprint of the 1968 original. MR.1484415 (98g:11066) 
[ARG] Arithmetic geometry, Springer-Verlag, New York, 1986. Papers from the conference held at the University of Connecticut, Storrs, Connecticut, July 30-August 10, 1984; Edited by Gary Cornell and Joseph H. Silverman. MR861969 (89b:14029)

[BAL] N. Bourbaki, Algébre, Hermann, Paris, 1958.

[BDJ] Kevin Buzzard, Fred Diamond, and Frazer Jarvis, On Serre's conjecture for mod $\ell$ Galois representations over totally real fields, Duke Math. J. 155 (2010), no. 1, 105-161, DOI 10.1215/00127094-2010-052. MR2730374 (2012k:11067)

[BGK] G. Banaszak, W. Gajda, and P. Krasoń, On Galois representations for abelian varieties with complex and real multiplications, J. Number Theory 100 (2003), no. 1, 117-132, DOI 10.1016/S0022-314X(02)00121-X. MR1971250 (2004a:11042)

[BL] Siegfried Bosch and Werner Lütkebohmert, Formal and rigid geometry. I. Rigid spaces, Math. Ann. 295 (1993), no. 2, 291-317, DOI 10.1007/BF01444889. MR.1202394 (94a:11090)

[C] Brian Conrad, Several approaches to non-Archimedean geometry, p-adic geometry, Univ. Lecture Ser., vol. 45, Amer. Math. Soc., Providence, RI, 2008, pp. 9-63. MR.2482345 (2011a:14047)

[dJ] A. J. de Jong, Crystalline Dieudonné module theory via formal and rigid geometry, Inst. Hautes Études Sci. Publ. Math. 82 (1995), 5-96 (1996). MR.1383213 (97f:14047)

[dJ1] A. J. De Jong, Erratum to: "Crystalline Dieudonné module theory via formal and rigid geometry" Inst. Hautes Études Sci. Publ. Math. No. 87 (1998), 175.

[DLS] Sigurdur Helgason, Differential geometry, Lie groups, and symmetric spaces, Pure and Applied Mathematics, vol. 80, Academic Press Inc. [Harcourt Brace Jovanovich Publishers], New York, 1978. MR514561 (80k:53081)

[E] M. Emerton, A $p$-adic variational Hodge conjecture and modular forms with complex multiplication, preprint

[EAI] H. Hida, Elliptic Curves and Arithmetic Invariants, Springer Monographs in Mathematics, to be published in 2013.

[EGA] A. Grothendieck and J. Dieudonné, Eléments de Géométrie Algébrique, Publications IHES 4 (1960), 8 (1961), 11 (1961), 17 (1963), 20 (1964), 24 (1965), 28 (1966), 32 (1967).

[GIT] David Mumford, Geometric invariant theory, Ergebnisse der Mathematik und ihrer Grenzgebiete, Neue Folge, Band 34, Springer-Verlag, Berlin, 1965. MR0214602 (35 \#5451)

[G] Eknath Ghate, Ordinary forms and their local Galois representations, Algebra and number theory, Hindustan Book Agency, Delhi, 2005, pp. 226-242. MR2193355 (2006i:11056)

[GV] Eknath Ghate and Vinayak Vatsal, On the local behaviour of ordinary $\Lambda$-adic representations, Ann. Inst. Fourier (Grenoble) 54 (2004), no. 7, 2143-2162 (2005) (English, with English and French summaries). MR2139691 (2006b:11050)

[GV1] B. Balasubramanyam, E. Ghate and V. Vatsal, On local Galois representations attached to ordinary Hilbert modular forms, preprint 2012

[GME] Haruzo Hida, Geometric modular forms and elliptic curves, 2nd ed., World Scientific Publishing Co. Pte. Ltd., Hackensack, NJ, 2012. MR2894984 (2012j:11100)

[H06] Haruzo Hida, Automorphism groups of Shimura varieties, Doc. Math. 11 (2006), 25-56 (electronic). MR2226268 (2007e:11067)

[H10] Haruzo Hida, The Iwasawa $\mu$-invariant of p-adic Hecke L-functions, Ann. of Math. (2) 172 (2010), no. 1, 41-137, DOI 10.4007/annals.2010.172.41. MR.2680417 (2012d:11215)

[H11a] Haruzo Hida, Constancy of adjoint $\mathcal{L}$-invariant, J. Number Theory 131 (2011), no. 7, 1331-1346, DOI 10.1016/j.jnt.2011.02.001. MR2782844 (2012k:11071)

[H11b] Haruzo Hida, Irreducibility of the Igusa tower over unitary Shimura varieties, On certain $L$-functions, Clay Math. Proc., vol. 13, Amer. Math. Soc., Providence, RI, 2011, pp. 187203. MR2767517(2012c:11131)

[IAT] Goro Shimura, Introduction to the arithmetic theory of automorphic functions, Publications of the Mathematical Society of Japan, No. 11. Iwanami Shoten, Publishers, Tokyo, 1971. Kanô Memorial Lectures, No. 1. MR0314766 (47 \#3318)

[K] N. Katz, Serre-Tate local moduli, Algebraic surfaces (Orsay, 1976), Lecture Notes in Math., vol. 868, Springer, Berlin, 1981, pp. 138-202. MR638600(83k:14039b)

[K1] Nicholas M. Katz, p-adic L-functions for CM fields, Invent. Math. 49 (1978), no. 3, 199297, DOI 10.1007/BF01390187. MR.513095 (80h:10039)

[M] Toshitsune Miyake, On automorphism groups of the fields of automorphic functions, Ann. of Math. (2) 95 (1972), 243-252. MR0300977 (46 \#137) 
[N] Rutger Noot, Abelian varieties - Galois representation and properties of ordinary reduction, Compositio Math. 97 (1995), no. 1-2, 161-171. Special issue in honour of Frans Oort. MR.1355123 (97a:11093)

[NAA] S. Bosch, U. Güntzer, and R. Remmert, Non-Archimedean analysis, Grundlehren der Mathematischen Wissenschaften [Fundamental Principles of Mathematical Sciences], vol. 261, Springer-Verlag, Berlin, 1984. A systematic approach to rigid analytic geometry. MR746961 (86b:32031)

[O] A. Ogus, Hodge cycles and crystalline cohomology, in: Hodge Cycles, Motives, and Shimura Varieties, Chapter VI, Lecture Notes in Math. 900 (1982), 357-414.

[PAF] Haruzo Hida, p-adic automorphic forms on Shimura varieties, Springer Monographs in Mathematics, Springer-Verlag, New York, 2004. MR2055355 (2005e:11054)

[Ra] M. Rapoport, Compactifications de l'espace de modules de Hilbert-Blumenthal, Compositio Math. 36 (1978), no. 3, 255-335 (French). MR515050 (80j:14009)

[Ri] Kenneth A. Ribet, Galois action on division points of Abelian varieties with real multiplications, Amer. J. Math. 98 (1976), no. 3, 751-804. MR0457455 (56 \#15660)

[Se] Jean-Pierre Serre, Quelques applications du théorème de densité de Chebotarev, Inst. Hautes Études Sci. Publ. Math. 54 (1981), 323-401 (French). MR644559 (83k:12011)

[Sh] Goro Shimura, On canonical models of arithmetic quotients of bounded symmetric domains, Ann. of Math. (2) 91 (1970), 144-222. MR0257031 (41 \#1686)

[T] J. T. Tate, $p$-divisible groups, Proc. Conf. Local Fields (Driebergen, 1966), Springer, Berlin, 1967, pp. 158-183. MR0231827 (38 \#155)

[Z] B. Zhao, Local indecomposability of Hilbert modular Galois representations, preprint, 2012 (posted in web: arXiv:1204.4007v1 [math.NT])

Department of Mathematics, University of California, Los Angeles, Los Angeles, CALIFORNIA 90095-1555

E-mail address: hida@math.ucla.edu 